\title{
Enriched Relative Polar Curves and Discriminants*
}

\author{
David B. Massey
}

\begin{abstract}
Let $(f, g)$ be a pair of complex analytic functions on a singular analytic space $X$. We give "the correct" definition of the relative polar curve of $(f, g)$, and we give a very formal generalization of Lê's attaching result, which relates the relative polar curve to the relative cohomology of the Milnor fiber modulo a hyperplane slice. We also give the technical arguments which allow one to work with a derived category version of the discriminant and Cerf diagram of a pair of functions. From this, we derive a number of generalizations of results which are classically proved using the discriminant.
\end{abstract}

\section{Lê's Attaching Result and Our Previous Generalization}

Let $\mathcal{U}$ be an open neighborhood of the origin in $\mathbb{C}^{n+1}$, and let $\tilde{f}: \mathcal{U} \rightarrow \mathbb{C}$ be a complex analytic function. We assume that $\mathbf{0} \in V(\tilde{f}):=\tilde{f}^{-1}(0)$. We let $\Sigma \tilde{f}$ denote the critical locus of $\tilde{f}$.

In this paper, we describe an improvement/generalization of what is now a classic result in the study of singularities: the attaching result of Lê in [7], which tells one how many $n$-cells are attached, up to homotopy, to a hyperplane slice of the Milnor fiber of $\tilde{f}$ in order to obtain the Milnor fiber, $F_{\tilde{f}, \mathbf{0}}$, of $\tilde{f}$ itself.

However, first, we must discuss the relative polar curve.

Fix a point $p \in \mathcal{U}$. Let $z_{0}$ denote a generic linear form on $\mathbb{C}^{n+1}$, which, in fact, we take as the first coordinate function, after possibly performing a generic linear change of coordinates.

In [4], 22, 17, 8], Hamm, Teissier, and Lê define and use the relative polar curve (of $\tilde{f}$ with respect to $\left.z_{0}\right), \Gamma_{\tilde{f}, z_{0}}^{1}$, to prove a number of topological results related to the Milnor fiber of hypersurface singularities. We shall recall some definitions and results here. We should mention that there are a number of different characterizations of the relative polar, all of which agree when $z_{0}$ is sufficiently generic; below, we have selected what we consider the easiest way of describing the relative polar curve as a set, a scheme, and a cycle.

As a set, $\Gamma_{\tilde{f}, z_{0}}^{1}$ is the closure of the critical locus of $\left(\tilde{f}, z_{0}\right)$ minus the critical locus of $\tilde{f}$, i.e., $\Gamma_{\tilde{f}, z_{0}}^{1}$ equals $\overline{\Sigma\left(\tilde{f}, z_{0}\right)-\Sigma \tilde{f}}$, as a set. If $z_{0}$ is sufficiently generic for $\tilde{f}$ at $p$, then, in a neighborhood of $p, \Gamma_{\tilde{f}, z_{0}}^{1}$ will be purely one-dimensional (which includes the possibility of being empty); see Theorem 1.1 below.

It is not difficult to give $\Gamma_{\tilde{f}, z_{0}}^{1}$ a scheme structure. We use $\left(z_{0}, \ldots, z_{n}\right)$ as coordinates on $\mathcal{U}$. If $\Gamma_{\tilde{f}, z_{0}}^{1}$ is purely one-dimensional at $p$, then, at points $x$ near, but unequal to, $p, \Gamma_{\tilde{f}, z_{0}}^{1}$ is given the structure of the

* AMS subject classifications 32B15, 32C35, 32 C18, 32B10.

keywords: polar curve, discriminant, Milnor fiber, nearby cycles. 
scheme $V\left(\frac{\partial \tilde{f}}{\partial z_{1}}, \ldots, \frac{\partial \tilde{f}}{\partial z_{n}}\right)$. One can also remove "algebraically" any embedded components of $\Gamma_{\tilde{f}, z_{0}}^{1}$ at $p$ by using gap sheaves; see Chapter 1 of [12].

In practice, all topological applications of the relative polar curve use only its structure as an analytic cycle (germ), that is, as a locally finite sum of irreducible analytic sets (or germs of sets) counted with integral multiplicities (which will all be non-negative). We remark here that these are cycles, not cycle classes; we do not mean up to rational equivalence. The intersection theory that one needs here is the simple case of proper intersections inside smooth manifolds; see 8.2 of [2] or our summary in Appendix A of [16]. If $C$ is a one-dimensional irreducible germ of $\Gamma_{\tilde{f}, z_{0}}^{1}$ at $p$, and $x \in C$ is close to, but unequal to, $p$, then the component $C$ appears in the cycle $\Gamma_{\tilde{f}, z_{0}}^{1}$ with multiplicity given by the Milnor number of $\tilde{f}_{\left.\right|_{H}}$ at $x$, where $H$ is a generic affine hyperplane passing through $x$.

The following theorem tells one the relative polar curve has nice properties for a generic choice of the linear form $z_{0}$.

Theorem 1.1.(Hamm-Lê) For a generic choice of $z_{0}$,

1. $\Gamma_{\tilde{f}, z_{0}}^{1}$ is purely one-dimensional at $p$;

2. $\Gamma_{\tilde{f}, z_{0}}^{1}$ properly intersects $V(\tilde{f}-\tilde{f}(p))$ at $p$, i.e., $p$ is an isolated point in $\Gamma_{\tilde{f}, z_{0}}^{1} \cap V(\tilde{f}-\tilde{f}(p))$;

3. the cycle $\Gamma_{\tilde{f}, z_{0}}^{1}$ is reduced (near $p$ ), i.e., each component through p appears with multiplicity 1.

Proof. Items 1 and 2 are proved in 2.1 of [4]. Item 3 is Lemma 2.2.1 of [4].

Now, we can state the main result of [7].

Theorem 1.2. (Lê, []) For generic $z_{0}$, up to homotopy, $F_{\tilde{f}, \mathbf{0}}$ is obtained from $F_{\tilde{f}_{\left.\right|_{\left(z_{0}\right)}}, \mathbf{0}}$ by attaching $\tau:=\left(\Gamma_{\tilde{f}, z_{0}}^{1} \cdot V(f)\right)_{\mathbf{0}} n$-cells.

In particular, $H^{k}\left(F_{\tilde{f}, \mathbf{0}}, F_{\tilde{f}_{\left.\right|_{V\left(z_{0}\right)}}, \mathbf{0}}\right)=0$ if $k \neq n$, and $H^{n}\left(F_{\tilde{f}, \mathbf{0}}, F_{\tilde{f}_{\mid V\left(z_{0}\right)}}, \mathbf{0}\right) \cong \mathbb{Z}^{\tau}$.

Remark 1.3. It will be important for us to understand some of Lê's set-up in [7].

Let $B_{\epsilon}$ denote a closed $2 n$-ball of radius $\epsilon$ centered at the origin in $\mathbb{C}^{n}$, and let $\mathbb{D}_{\delta}$ denote a closed disk of radius $\delta$ centered at the origin in $\mathbb{C}$.

Then, Lê shows that, for $0<|\xi| \ll \delta \ll \epsilon \ll 1,\left(\mathbb{D}_{\delta} \times B_{\epsilon}\right) \cap \tilde{f}^{-1}(\xi)$ has the homotopy-type of the Milnor fiber of $\tilde{f}$ at $\mathbf{0}$ and, of course, $\left(\{0\} \times B_{\epsilon}\right) \cap \tilde{f}^{-1}(\xi)$ is homeomorphic to the Milnor fiber, $F_{\tilde{f}_{0}, \mathbf{0}}$, of $\tilde{f}_{0}:=\tilde{f}_{\left.\right|_{V\left(z_{0}\right)}}$ at $\mathbf{0}$. Theorem 1.2 is obtained by applying Morse Theory to the map $\left|z_{0}\right|^{2}$ on $\left(\mathbb{D}_{\delta} \times B_{\epsilon}\right) \cap f^{-1}(\xi)$.

In [7], Lê gives an extensive discussion of the discriminant and Cerf diagram of the map $G:=\left(z_{0}, \tilde{f}\right)$. The discriminant is $G(\Sigma G)$ and the Cerf diagram is $G\left(\Gamma_{\tilde{f}, z_{0}}^{1}\right)$. One "sees" Lê's attaching result graphically in the discriminant/Cerf diagram below. Down in the image of the map $\left(z_{0}, \tilde{f}\right)$, the pair $\left(F_{\tilde{f}, \mathbf{0}}, F_{\tilde{f}_{0}, \mathbf{0}}\right)$ is represented by the pair $(L,\{a\})$, and one sees that the relative cohomology of $\left(F_{\tilde{f}, \mathbf{0}}, F_{\tilde{f}_{0}, \mathbf{0}}\right)$ decomposes as a direct sum of local Morse data above each of the points where $L$ intersects the Cerf diagram, $C$, (the image of the relative polar curve). Now, the number of points in the intersection of $C$ and $L$, counted with multiplicities, is precisely $\tau=\left(\Gamma_{\tilde{f}, z_{0}}^{1} \cdot V(f)\right)_{0}$, which yields the theorem. 


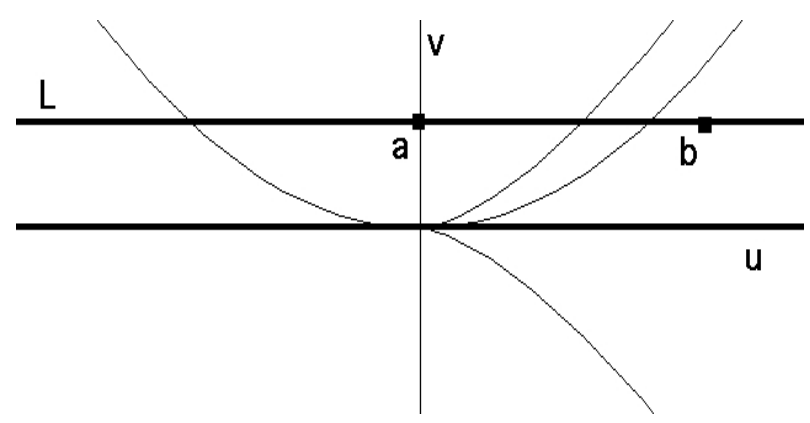

From Lê's discussion, it is clear that $F_{\tilde{f}_{0}, \mathbf{0}}$ is also homeomorphic to $\widehat{F}_{\tilde{f}_{0}, \mathbf{0}}:=\left(\{\nu\} \times B_{\epsilon}\right) \cap \tilde{f}^{-1}(\xi)$, provided that $0<|\xi| \ll \nu<\delta \ll \epsilon \ll 1$; in fact, there is a homeomorphism from the pair $\left(F_{\tilde{f}, \mathbf{0}}, F_{\tilde{f}_{0}, \mathbf{0}}\right)$ to $\left(F_{\tilde{f}, \mathbf{0}}, \widehat{F}_{\tilde{f}_{0}, \mathbf{0}}\right)$ which induces an isomorphism between $H^{*}\left(F_{\tilde{f}, \mathbf{0}}, F_{\tilde{f}_{0}, \mathbf{0}}\right)$ and $H^{*}\left(F_{\tilde{f}, \mathbf{0}}, \widehat{F}_{\tilde{f}_{0}, \mathbf{0}}\right)$ and which induces the identity map on $H^{*}\left(F_{\tilde{f}, \mathbf{0}}\right)$. In the Cerf diagram, the pair $\left(F_{\tilde{f}, \mathbf{0}}, \widehat{F}_{\tilde{f}_{0}, \mathbf{0}}\right)$ is represented by $(L,\{b\})$ and, using an argument which is essentially the same as in the paragraph above, one concludes that $H^{*}\left(F_{\tilde{f}, \mathbf{0}}, \widehat{F}_{\tilde{f}_{0}, \mathbf{0}}\right) \cong \mathbb{Z}^{\tau}$.

This is an important observation, because in the formalism of the derived category and vanishing cycles, $H^{k+1}\left(F_{\tilde{f}, \mathbf{0}}, \widehat{F}_{\tilde{f}_{0}, \mathbf{0}}\right)$ is isomorphic to the stalk cohomology at the origin of the vanishing cycles along $z_{0}$ of the nearby cycles along $f$ of the constant sheaf on $\mathcal{U}$, i.e., $H^{k+1}\left(F_{\tilde{f}, \mathbf{0}}, \widehat{F}_{\tilde{f}_{0}, \mathbf{0}}\right) \cong H^{k}\left(\phi_{z_{0}} \psi_{f} \mathbb{Z}_{\mathcal{U}}\right)_{\mathbf{0}}$ (here, we do not distinguish between $z_{0}$ and $\left.z_{\left.0\right|_{V(f)}}\right)$. If we include the correct shifts, then we know that $\mathbb{Z}_{\mathcal{U}}[n+1]$ is perverse, and that $\phi_{z_{0}}[-1]$ and $\psi_{f}[-1]$ take perverse sheaves to perverse sheaves; hence, we prefer to write $H^{k}\left(\phi_{z_{0}}[-1] \psi_{f}[-1] \mathbb{Z}_{\mathcal{U}}[n+1]\right)_{\mathbf{0}} \cong H^{k+n}\left(F_{\tilde{f}, \mathbf{0}}, \widehat{F}_{\tilde{f}_{0}, \mathbf{0}}\right)$.

Thus, the results of Lê in [7] tell one that $H^{k}\left(\phi_{z_{0}}[-1] \psi_{f}[-1] \mathbb{Z}_{\mathcal{U}}^{\bullet}[n+1]\right)_{\mathbf{0}}$ is zero, unless $k=0$, and $H^{0}\left(\phi_{z_{0}}[-1] \psi_{f}[-1] \mathbb{Z}_{\mathcal{U}}^{\bullet}[n+1]\right)_{\mathbf{0}} \cong \mathbb{Z}^{\tau}$.

The above relation between Theorem 1.2 and iterated vanishing and nearby cycles appeared explicitly in the work of Sabbah in 21] and in our own work in [13].

\section{Summary of our Old Results from 13 .}

We now wish to describe one of our primary results from [13], which is a substantial generalization of Theorem 1.2 but first we need recall some of our previous definitions. One of our main goals in the current paper is to replace these old definitions with more natural ones.

Let $X$ be a closed analytic subspace of $\mathcal{U}$, and let $f:=\tilde{f}_{\mid X}$.

A good stratification of $X$ relative to $f$ is a complex analytic stratification $\mathfrak{S}$ of $X$ such that all of the strata $S \in \mathfrak{S}$ are connected, $V(f)$ is a union of strata, $\mathfrak{S}^{o}:=\{S \in \mathfrak{S} \mid S \nsubseteq V(f)\}$ is a Whitney stratification of $X-V(f)$ and such that, for every pair of strata $\left(S_{\alpha}, S_{\beta}\right)$ such that $S_{\alpha} \nsubseteq \subseteq V(f)$ and $S_{\beta} \subseteq V(f)$, Thom's $a_{f}$ condition is satisfied. In our setting this is equivalent to: if $\mathbf{p} \in S_{\beta} \subseteq V(f)$ and $\mathbf{p}_{i} \in S_{\alpha} \nsubseteq V(f)$ are such that $\mathbf{p}_{i} \rightarrow \mathbf{p}$ and $T_{\mathbf{p}_{i}} V\left(f_{\left.\right|_{S_{\alpha}}}-f_{\left.\right|_{S_{\alpha}}}\left(\mathbf{p}_{i}\right)\right)$ converges to some $\mathcal{T}$, then $T_{\mathbf{p}} S_{\beta} \subseteq \mathcal{T}$; note that this implies that the pair $\left(S_{\alpha}, S_{\beta}\right)$ must satisfy Whitney's condition (a). In a good stratification, we call the strata which comprise $V(f)$ the good strata; we refer to the other strata as outside strata. Note that we do not require that Whitney's condition (b) hold along good strata, or even that Whitney's condition (a) holds between pairs of good strata.

Fix a good stratification $\mathfrak{S}$ for $X$ relative to $f$. 
Let $\tilde{g}:(\mathcal{U}, \mathbf{0}) \rightarrow(\mathbb{C}, 0)$ be another analytic function, and let $g:=\tilde{g}_{\left.\right|_{X}}$. If $Y$ is an analytic subset of $X$, we define $\Gamma_{f, g}(Y)$ to be the closure in $X$ of the critical locus of $\Phi_{\left.\right|_{Y-\Sigma Y-V(f)}}$. This is called the relative polar variety of $Y$ with respect to $f$ and $g$

For each $S \in \mathfrak{S}, \Gamma_{f, g}(S)$ is thus the closure of the critical locus of $(f, g)_{\left.\right|_{S-V(f)}}$. The union $\cup_{S \in \mathfrak{S} \Gamma_{f, g}}(S)$ is called the relative polar variety of $f$ and $g$ with respect to $\mathfrak{S}$, and we denote it by $\Gamma_{f, g}(\mathfrak{S})$ (or, simply, $\Gamma_{f, g}$ if the stratification is clear). Note that if $S \subseteq V(f)$, then $\Gamma_{f, g}(S)=\emptyset$.

For each stratum $S \in \mathfrak{S}$, we define the symmetric relative polar variety of $S$ with respect to $f$ and $g, \widetilde{\Gamma}_{f, g}(S)$, to be the closure in $X$ of the critical locus of $(f, g)_{\left.\right|_{S-V(f)-V(g)}}$. We also define the symmetric relative polar variety of $f$ and $g$ with respect to $\mathfrak{S}, \widetilde{\Gamma}_{f, g}(\mathfrak{S})$, to be the union $\bigcup_{S \in \mathfrak{S}} \widetilde{\Gamma}_{f, g}(S)$. We use the term "symmetric" since we obviously have $\widetilde{\Gamma}_{f, g}(Y)=\widetilde{\Gamma}_{g, f}(Y)$.

In the special case where $f$ and $g$ are such that $\widetilde{\Gamma}_{f, g}(\mathfrak{S})$ is one-dimensional, we naturally refer to the symmetric polar variety as the symmetric polar curve and emphasize the fact that it is one- dimensional by writing $\widetilde{\Gamma}_{f, g}^{1}(\mathfrak{S})$. In this case, we wish to give the symmetric polar curve the structure of a cycle (actually, a cycle germ at the origin), so we must attach some multiplicity to each component of this curve.

To do this, for each (one-dimensional) component, $\nu$, of $\widetilde{\Gamma}_{f, g}^{1}(\mathfrak{S})$, let $S_{\nu}$ denote the stratum which contains $\nu-\mathbf{0}$ near the origin. If $S_{\nu}$ is itself one-dimensional, we assign the multiplicity 1 to $\nu$ (that is, we consider $\nu$ with its reduced structure). Now, to each $\nu$ for which $S_{\nu}$ is not one-dimensional, we assign the multiplicity given by the Milnor number of the map $g$ restricted to $S_{\nu} \cap V(f-f(\mathbf{p}))$ at any point $\mathbf{p} \in \nu-\mathbf{0}$ sufficiently close to the origin. We use here that $S_{\nu} \cap V(f-f(\mathbf{p}))$ is a manifold at $\mathbf{p}$, and that $g$ restricted to this set has an isolated critical point at $\mathbf{p}$ since $\nu$ is one-dimensional.

The function $g$ is tractable at the origin with respect to a good stratification $\mathfrak{S}$ of $X$ relative to $f:(X, \mathbf{0}) \rightarrow$ $(\mathbb{C}, 0)$ if and only if $\operatorname{dim}_{0} \widetilde{\Gamma}_{f, g}^{1}(\mathfrak{S}) \leq 1$ and, for all good strata $S_{\alpha}, g_{\left.\right|_{S_{\alpha}}}$ has no critical points in a neighborhood of the origin except, perhaps, at the origin itself.

We say that $g$ is decent with respect to $\mathfrak{S}$ relative to $f$ provided that $d_{\mathbf{p}} \tilde{g}$ is not a degenerate covector (see [3]) at any stratified critical point of $g$ restricted to $F_{f, 0}-V(g)$ (with respect to the induced stratification on $\left.F_{f, \mathbf{0}}\right)$. See Section 1 of [13. Note that this condition is automatic if $\mathfrak{S}$ has only one stratum not contained in $V(f) \cup V(g)$.

By combining Proposition 1.12 and 1.14 of [13], we obtain:

Proposition 1.4. Let $\mathfrak{S}$ be a good stratification of $X$ for $f$ at the origin. Then, for a generic choice of linear forms, $l, l$ is decent and tractable with respect to $\mathfrak{S}$ relative to $f$.

For $S \in \mathfrak{S}^{o}$, we let $\mathbb{N}_{S}$ and $\mathbb{L}_{S}$ denote, respectively, the normal slice and link of the stratum $S$; see [3]. In [13, we proved:

Theorem 1.5. (13], Theorem 4.2) Suppose that $g$ is tractable relative to $f$ with respect to a good stratification $\mathfrak{S}$ of $X$ at $\mathbf{0}$. Let $d_{S}$ denote the dimension of $S \in \mathfrak{S}$. Let $\mathbf{F}^{\bullet}$ be a bounded complex of sheaves of $\mathbb{Z}$-modules on $X-V(f)$, constructible with respect to $\mathfrak{S}^{\circ}$.

Then, for all $i, \mathbb{H}^{i}\left(F_{f, \mathbf{0}}, F_{f_{\left.\right|_{V(g)}}}, \mathbf{0} ; \mathbf{F}^{\bullet}\right)$ is a direct summand of $H^{i-1}\left(\phi_{g} \psi_{f} \mathbf{F}^{\bullet}\right)_{\mathbf{0}}$, and there exist integers 
$j_{S}$ such that

$$
\mathbb{H}^{i}\left(F_{f, \mathbf{0}}, F_{f_{\mid V(g)}}, \mathbf{0} ; \mathbf{F}^{\bullet}\right) \cong \bigoplus_{S \in \mathfrak{S}^{\circ}}\left(\mathbb{H}^{i-d_{S}+1}\left(\mathbb{N}_{S}, \mathbb{L}_{S} ; \mathbf{F}^{\bullet}\right)\right)^{j_{S}}
$$

where $j_{S} \geq\left(\widetilde{\Gamma}_{f, g}^{1}(S) \cdot V(f)\right)_{\mathbf{0}}$, with equality if $g$ is decent relative to $f$.

Furthermore, if $\Gamma_{f, g}(\mathfrak{S})$ has no components contained in $V(g)$ (i.e., if $\Gamma_{f, g}(\mathfrak{S})=\widetilde{\Gamma}_{f, g}(\mathfrak{S})$ ), then

$$
H^{i-1}\left(\phi_{g} \psi_{f} \mathbf{F}^{\bullet}\right)_{\mathbf{0}} \cong \mathbb{H}^{i}\left(F_{f, \mathbf{0}}, F_{f_{\left.\right|_{V(g)}}, \mathbf{0}} ; \mathbf{F}^{\bullet}\right) \text {. }
$$

\section{Summary of the Results of this Paper}

What are the problems with Theorem 1.5. There are several. One is that the hypotheses are difficult to check. Another, related, problem is that it is unclear to what extent the hypotheses are necessary for the conclusion. A third issue is that the definition "relative symmetric polar curve" seems rather ad hoc.

In this paper, we "fix" these problems. Let $\mathfrak{S}^{o}\left(\mathbf{F}^{\bullet}\right)$ be the set of strata of $\mathfrak{S}$ such that $\mathbb{H}^{*}\left(\mathbb{N}_{S}, \mathbb{L}_{S} ; \mathbf{F}^{\bullet}\right) \neq 0$ and $f_{\left.\right|_{S}}$ is not constant. For each $S \in \mathfrak{S}^{\circ}\left(\mathbf{F}^{\bullet}\right)$, we will define an (ordinary) cycle $\Gamma_{f, \tilde{g}}(S)$. Using these cycles, we will define (Definition 13.4) the graded, enriched relative polar cycle, $\left(\Gamma_{f, \tilde{g}}\left(\mathbf{F}^{\bullet}\right)\right)^{\bullet}$. In each degree $k \in \mathbb{Z},\left(\Gamma_{f, \tilde{g}}\left(\mathbf{F}^{\bullet}\right)\right)^{k}$ is a formal, locally finite, sum of irreducible analytic subsets of $X$ multiplied by modules over a fixed base ring; see Section 2 of [17] and Section 3. When the underlying set, $\left|\left(\Gamma_{f, \tilde{g}}\left(\mathbf{F}^{\bullet}\right)\right)^{\bullet}\right|=$ $\bigcup_{S \in \mathfrak{S}^{\circ}\left(\mathbf{F}^{\bullet}\right)}\left|\Gamma_{f, \tilde{g}}(S)\right|$ is purely one-dimensional at a point $p \in X$, we say that the relative polar curve of $f$, with respect to $g$, with coefficients in $\mathbf{F}^{\bullet}$, is defined at $p$. A principal theme of this paper is that this definition of the relative polar curve is the correct definition in results on the cohomology level.

The intersection product that we use throughout our work is a mild extension of the intersection theory, mentioned above, of properly intersecting cycles in a complex manifold; see Section 2 of [17] for the fundamental properties. We use $\odot$ to denote this enriched intersection product.

Using our results in [17, and continuing with the notation from above, we will quickly prove our first main theorem:

Main Theorem 1. (Theorem 3.11) In a neighborhood of the origin,

$$
\operatorname{supp} \phi_{g}[-1] \psi_{f}[-1] \mathbf{F}^{\bullet}=V(f) \cap\left|\Gamma_{f, \tilde{g}}\left(\mathbf{F}^{\bullet}\right)\right| \subseteq V(g),
$$

and, when $\operatorname{dim}_{\mathbf{0}} V(f) \cap\left|\Gamma_{f, \tilde{g}}\left(\mathbf{F}^{\bullet}\right)\right| \leq 0$,

$$
H^{k}\left(\phi_{g}[-1] \psi_{f}[-1] \mathbf{F}^{\bullet}\right)_{\mathbf{0}} \cong\left(\left(\Gamma_{f, \tilde{g}}\left(\mathbf{F}^{\bullet}\right)\right)^{k} \odot V(f)\right)_{\mathbf{0}},
$$

i.e.,

$$
H^{k-1}\left(B_{\epsilon} \cap f^{-1}(\xi), B_{\epsilon} \cap f^{-1}(\xi) \cap g^{-1}(\nu) ; \mathbf{F}^{\bullet}\right) \cong \bigoplus_{S \in \mathfrak{S}^{\circ}\left(\mathbf{F}^{\bullet}\right)}\left(\mathbb{H}^{k-d_{S}}\left(\mathbb{N}_{S}, \mathbb{L}_{S} ; \mathbf{F}^{\bullet}\right)\right)^{j_{S}},
$$

where $0<|\xi| \ll|\nu|<\ll \epsilon \ll 1$, and $j_{S}=\left(\Gamma_{f, g}^{1}(S) \cdot V(f)\right)_{\mathbf{0}}$.

Our proof of the above theorem is elegant, and very short, given existing results. However, it is not as intuitive as the discriminant/Cerf diagram argument, nor does it allow us to prove a number of related results, as we did in Section 4 of [13. 
Hence, in Section 4 of this paper, we will prove the necessary technical results to push-down the complex $\mathbf{F}^{\bullet}$, restricted to a suitable neighborhood, via the map $(g, f)$. This will give us a derived category version of the discriminant and Cerf diagram, in which the standard intuitive proofs work without modification. Somewhat surprisingly, the hypothesis that we need is precisely that of Main Theorem 1.

We prove:

Main Theorem 2. (Theorem 4.13) Suppose that $\operatorname{dim}_{\mathbf{0}} V(f) \cap\left|\Gamma_{f, \tilde{g}}\left(\mathbf{F}^{\bullet}\right)\right| \leq 0$. Let $\left(\widehat{\Gamma}_{f, \tilde{g}}\left(\mathbf{F}^{\bullet}\right)\right)^{\bullet}$ denote the components of $\left(\Gamma_{f, \tilde{g}}\left(\mathbf{F}^{\bullet}\right)\right)^{\bullet}$ which are not contained in $V(g)$. Then,

1.

$$
\mathbb{H}^{k-1}\left(F_{f, \mathbf{0}}, F_{f_{\left.\right|_{V(g)}}, \mathbf{0}} ; \mathbf{F}^{\bullet}\right) \cong\left(\left(\widehat{\Gamma}_{f, \tilde{g}}\left(\mathbf{F}^{\bullet}\right)\right)^{k} \odot V(f)\right)_{\mathbf{0}}
$$

2.

$$
\mathbb{H}^{k-1}\left(F_{g, \mathbf{0}}, F_{g_{\left.\right|_{V}(f)}, \mathbf{0}} ; \mathbf{F}^{\bullet}\right) \cong\left(\left(\widehat{\Gamma}_{f, \tilde{g}}\left(\mathbf{F}^{\bullet}\right)\right)^{k} \odot V(g)\right)_{\mathbf{0}} ; \text { and }
$$

3.

$$
H^{k}\left(\phi_{f}[-1] \psi_{g}[-1] \mathbf{F}^{\bullet}\right)_{\mathbf{0}} \cong\left(\left(\widehat{\Gamma}_{f, \tilde{g}}\left(\mathbf{F}^{\bullet}\right)\right)^{k} \odot V(g)\right)_{\mathbf{0}} \oplus H^{k}\left(\psi_{g}[-1] \phi_{f}[-1] \mathbf{F}^{\bullet}\right)_{\mathbf{0}}
$$

In Section 5 we will combine the results of Section 4 with Corollary 3.9 of [18 in order to obtain a relation between Thom's $a_{f}$ condition and the graded, enriched polar curve.

We belatedly thank Marc Levine for a number of helpful discussions involving our enriched intersection theory. We also thank Lê Dũng Tráng for some very helpful proofreading, and for a number of suggestions which improved the presentation.

\section{Basics of Enriched Cycles}

In this section, we will recall the basic definitions that one needs for using enriched cycles ; these definitions are taken from Section 2 of [17. There are a number of results from [17] which will be used in the proof of the main theorem in Section 3. While we will not restate the needed results from [17] in this paper, the background material in this section will enable the reader to make sense of the definition of the graded, enriched relative polar curve and the proof of the main theorem in Section 3

Definition 2.1. An enriched cycle, $E$, in $X$ is a formal, locally finite sum $\sum_{V} E_{V}[V]$, where the $V$ 's are irreducible analytic subsets of $X$ and the $E_{V}$ 's are finitely-generated $R$-modules. We refer to the $V$ 's as the components of $E$, and to $E_{V}$ as the $V$-component module of $E$. Two enriched cycles are considered the same provided that all of the component modules are isomorphic. The underlying set of $E$ is $|E|:=\cup_{E_{V} \neq 0} V$.

If $C=\sum n_{V}[V]$ is an ordinary positive cycle in $X$, i.e., all of the $n_{v}$ are non-negative integers, then there is a corresponding enriched cycle $[C]^{\mathrm{enr}}$ in which the $V$-component module is the free $R$-module of rank $n_{V}$. If $R$ is an integral domain, so that rank of an $R$-module is well-defined, then an enriched cycle $E$ yields an ordinary cycle $[E]^{\text {ord }}:=\sum_{V}\left(\operatorname{rk}\left(E_{V}\right)\right)[V]$.

If $q$ is a finitely-generated module and $E$ is an enriched cycle, then we let $q E:=\sum_{V}\left(q \otimes E_{V}\right)[V]$; thus, if $R$ is an integral domain and $E$ is an enriched cycle, $[q E]^{\text {ord }}=(\operatorname{rk}(q))[E]^{\text {ord }}$ and if $C$ is an ordinary positive cycle and $n$ is a positive integer, then $[n C]^{\mathrm{enr}}=R^{n}[C]^{\mathrm{enr}}$. 
The (direct) sum of two enriched cycles $D$ and $E$ is given by $(D+E)_{V}:=D_{V} \oplus E_{V}$.

There is a partial ordering on enriched cycles given by: $D \leq E$ if and only if there exists an enriched cycle $P$ such that $D+P=E$. This relation is clearly reflexive and transitive; moreover, anti-symmetry follows from the fact that if $M$ and $N$ are Noetherian modules such that $M \oplus N \cong M$, then $N=0$.

If two irreducible analytic subsets $V$ and $W$ intersect properly in $\mathcal{U}$, then the (ordinary) intersection cycle $[V] \cdot[W]$ is a well-defined positive cycle; we define the enriched intersection product of $[V]^{\mathrm{enr}}$ and $[W]^{\mathrm{enr}}$ by $[V]^{\mathrm{enr}} \odot[W]^{\mathrm{enr}}=([V] \cdot[W])^{\mathrm{enr}}$. If $D$ and $E$ are enriched cycles, and every component of $D$ properly intersects every component of $E$ in $\mathcal{U}$, then we say that $D$ and $E$ intersect properly in $\mathcal{U}$ and we extend the intersection product linearly, i.e., if $D=\sum_{V} D_{V}[V]$ and $E=\sum_{W} E_{W}[W]$, then

$$
D \odot E:=\sum_{V, W}\left(D_{V} \otimes E_{W}\right)([V] \cdot[W])^{\mathrm{enr}} .
$$

A graded, enriched cycle $E^{\bullet}$ is simply an enriched cycle $E^{i}$ for $i$ in some bounded set of integers. An single enriched cycle is considered as a graded enriched cycle by being placed totally in degree zero. The analytic set $V$ is a component of $E^{\bullet}$ if and only if $V$ is a component of $E^{i}$ for some $i$, and the underlying set of $E^{\bullet}$ is $\left|E^{\bullet}\right|=\cup_{i}\left|E^{i}\right|$. If $R$ is a domain, then $E^{\bullet}$ yields an ordinary cycle $\left[E^{\bullet}\right]^{\text {ord }}:=\sum_{i}(-1)^{i}\left(\operatorname{rk}\left(E_{V}^{i}\right)\right)[V]$. If $k$ is an integer, we define the $k$-shifted graded, enriched cycle $E^{\bullet}[k]$ by $\left(E^{\bullet}[k]\right)^{i}:=E^{i+k}$.

If $q$ is a finitely-generated module and $E^{\bullet}$ is a graded enriched cycle, then we define the graded enriched cycle $q E^{\bullet}$ by $\left(q E^{\bullet}\right)^{i}:=\sum_{V}\left(q \otimes E_{V}^{i}\right)[V]$. The (direct) sum of two graded enriched cycles $D^{\bullet}$ and $E^{\bullet}$ is given by $\left(D^{\bullet}+E^{\bullet}\right)_{V}^{i}:=D_{V}^{i} \oplus E_{V}^{i}$. If $D^{i}$ properly intersects $E^{j}$ for all $i$ and $j$, then we say that $D^{\bullet}$ and $E^{\bullet}$ intersect properly and we define the intersection product by

$$
\left(D^{\bullet} \odot E^{\bullet}\right)^{k}:=\sum_{i+j=k}\left(D^{i} \odot E^{j}\right) .
$$

Whenever we use the enriched intersection product symbol, we mean that we are considering the objects on both sides of $\odot$ as graded, enriched cycles, even if we do not superscript by enr or $\bullet$.

Let $\tau: W \rightarrow Y$ be a proper morphism between analytic spaces. If $C=\sum n_{V}[V]$ is an ordinary positive cycle in $W$, then the proper push-forward $\tau_{*}(C)=\sum n_{V} \tau_{*}([V])$ is a well-defined ordinary cycle.

Definition 2.2. If $E^{\bullet}=\sum_{V} E_{V}^{\bullet}[V]$ is an enriched cycle in $W$, then we define the proper push-forward of $E^{\bullet}$ by $\tau$ to be the graded enriched cycle $\tau_{*}^{\bullet}\left(E^{\bullet}\right)$ defined by

$$
\tau_{*}^{j}\left(E^{\bullet}\right):=\sum_{V} E_{V}^{j}\left[\tau_{*}([V])\right]^{\mathrm{enr}}
$$

The ordinary projection formula for divisors ([F], 2.3.c) immediately implies the following enriched version.

Proposition 2.3. Let $E^{\bullet}$ be a graded enriched cycle in $X$. Let $W:=\left|E^{\bullet}\right|$. Let $\tau: W \rightarrow Y$ be a proper morphism, and let $g: Y \rightarrow \mathbb{C}$ be an analytic function such that $g \circ \tau$ is not identically zero on any component of $E^{\bullet}$. Then, $g$ is not identically zero on any component of $\tau_{*}^{\bullet}\left(E^{\bullet}\right)$ and

$$
\tau_{*}^{\bullet}\left(E^{\bullet} \odot V(g \circ \tau)\right)=\tau_{*}^{\bullet}\left(E^{\bullet}\right) \odot V(g) .
$$


Definition 2.4. Suppose that $\mathbf{F}^{\bullet}$ is a bounded complex of sheaves, which is constructible with respect to an analytic Whitney stratification $\mathfrak{S}$, in which the strata are connected. For $S \in \mathfrak{S}$, let $d_{S}:=\operatorname{dim} S$. If $\left(\mathbb{N}_{S}, \mathbb{L}_{S}\right)$ is a pair consisting of a normal slice and complex link, respectively, to the stratum $S$, then, for each integer $k$, the isomorphism-type of the module $\mathbb{H}^{k-d_{S}}\left(\mathbb{N}_{S}, \mathbb{L}_{S} ; \mathbf{F}^{\bullet}\right)$ is independent of the choice of $\left(\mathbb{N}_{S}, \mathbb{L}_{S}\right)$; we refer to $\mathbb{H}^{k-d_{S}}\left(\mathbb{N}_{S}, \mathbb{L}_{S} ; \mathbf{F}^{\bullet}\right)$ as the degree $k$ Morse module of $S$ with respect to $\mathbf{F}^{\bullet}$.

The graded, enriched characteristic cycle of $\mathbf{F}^{\bullet}$ in the cotangent bundle $T^{*} \mathcal{U}$ is defined in degree $k$ to be

$$
\operatorname{gecc}^{k}\left(\mathbf{F}^{\bullet}\right):=\sum_{S \in \mathfrak{S}} H^{k-d_{S}}\left(\mathbb{N}_{S}, \mathbb{L}_{S} ; \mathbf{F}^{\bullet}\right)\left[\overline{T_{S}^{*} \mathcal{U}}\right]
$$

Remark 2.5. There are no canonical choices for defining the the normal slices or complex links of strata. However, as two enriched cycles are equal provided that the component modules are all isomorphic, the graded, enriched characteristic cycle is well-defined.

Example 2.6. We wish a give a simple example of calculating a graded, enriched characteristic cycle.

Let $f: \mathbb{C}^{3} \rightarrow \mathbb{C}$ be given by $f(x, y, t)=y\left(y^{2}-x^{3}-t^{2} x^{2}\right)$, and let $X:=V(f)=V(y) \cup V\left(y^{2}-x^{3}-t^{2} x^{2}\right)$. The singular set of $X, \Sigma X$, is the 1-dimensional set $V(x, y) \cup V\left(x+t^{2}, y\right)$. Thus, near the origin (actually, in this specific example, globally),

$$
\mathfrak{S}:=\left\{V(y)-V\left(y^{2}-x^{3}-t^{2} x^{2}\right), V\left(y^{2}-x^{3}-t^{2} x^{2}\right)-V(y), V(x, y)-\{\mathbf{0}\}, V\left(x+t^{2}, y\right)-\{\mathbf{0}\},\{\mathbf{0}\}\right\}
$$

is a Whitney stratification of $X$ with connected strata. Let $\mathbf{F}^{\bullet}:=\mathbb{Z}_{X}^{\bullet}[2]$ (we shall discuss the shift by 2 below), which is constructible with respect to any Whitney stratification of $X$. We wish to calculate $\operatorname{gecc}^{\bullet}\left(\mathbf{F}^{\bullet}\right)$.

First, consider the 2-dimensional strata. Let $S_{1}:=V(y)-V\left(y^{2}-x^{3}-t^{2} x^{2}\right)$. Then, $\mathbb{N}_{S_{1}}$ is simply a point, and $\mathbb{L}_{S_{1}}$ is empty. Hence, $H^{k-2}\left(\mathbb{N}_{S_{1}}, \mathbb{L}_{S_{1}} ; \mathbf{F}^{\bullet}\right)=H^{k}\left(\mathbb{N}_{S_{1}}, \mathbb{L}_{S_{1}} ; \mathbb{Z}\right)$ isomorphic to $\mathbb{Z}$ if $k=0$, and is 0 if $k \neq 0$. The same conclusion holds if $S_{1}$ is replaced by $S_{2}:=V\left(y^{2}-x^{3}-t^{2} x^{2}\right)-V(y)$.

Now, consider the 1-dimensional strata. Let $S_{3}:=V(x, y)-\{\mathbf{0}\}$, and $S_{4}:=V\left(x+t^{2}, y\right)-\{\mathbf{0}\}$. The normal slice $\mathbb{N}_{S_{3}}$ is, as a germ, up to analytic isomorphism, three complex lines in $\mathbb{C}^{2}$, which intersect at a point, and $\mathbb{L}_{S_{3}}$ is three points. Similarly, the normal slice $\mathbb{N}_{S_{4}}$ is, as a germ, up to analytic isomorphism, two complex lines in $\mathbb{C}^{2}$, which intersect at a point, and $\mathbb{L}_{S_{4}}$ is two points. Hence, $H^{k-1}\left(\mathbb{N}_{S_{3}}, \mathbb{L}_{S_{3}} ; \mathbf{F}^{\bullet}\right)=H^{k+1}\left(\mathbb{N}_{S_{3}}, \mathbb{L}_{S_{3}} ; \mathbb{Z}\right)$ isomorphic to $\mathbb{Z}^{2}$ if $k=0$, and is 0 if $k \neq 0$. Similarly, $H^{k-1}\left(\mathbb{N}_{S_{4}}, \mathbb{L}_{S_{4}} ; \mathbf{F}^{\bullet}\right)=H^{k+1}\left(\mathbb{N}_{S_{4}}, \mathbb{L}_{S_{4}} ; \mathbb{Z}\right)$ isomorphic to $\mathbb{Z}$ if $k=0$, and is 0 if $k \neq 0$.

Finally, consider the stratum $\{\mathbf{0}\}$. Then, $\mathbb{N}_{\{\mathbf{0}\}}$ is all of $X$, intersected with a small ball around the origin. The complex link $\mathbb{L}_{\{\mathbf{0}\}}$ is usually referred to as simply the complex link of $X$ at $\mathbf{0}$. Thus, $\mathbb{L}_{\{\mathbf{0}\}}$ has the homotopy-type of a bouquet of 1-spheres (see [10]), and the number of spheres in this bouquet is equal to the intersection number $\left(\Gamma_{f, L}^{1} \cdot V(L)\right)_{\mathbf{0}}$, where $L$ is any linear form such that $d_{\mathbf{0}} L$ is not a degenerate covector from strata of $X$ at 0 (see [3]), and the relative polar curve here is the classical one from the beginning of Section 11. We claim that we may use $L:=t$ for this calculation.

To see this, first note that $V\left(y^{2}-x^{3}-t^{2} x^{2}\right)$ is the classic example of a space such that the regular part satisfies Whitney's condition (a) along the $t$-axis (or, alternatively, this is an easy exercise). Thus, $d_{\mathbf{0}} t$ is not a limit of conormals from $S_{2}$. Now, the closures of $S_{1}, S_{3}$, and $S_{4}$ are all smooth, and $d_{0} t$ is not conormal to these closures at the origin. 
To find the ordinary cycle $\Gamma_{f, t}^{1}$, we take the components of the cycle below which are not contained in $\Sigma f$ :

$$
\begin{gathered}
V\left(\frac{\partial f}{\partial x}, \frac{\partial f}{\partial y}\right)=V\left(y\left(-3 x^{2}-2 t^{2} x\right), 3 y^{2}-x^{3}-t^{2} x^{2}\right)=V\left(y, x^{2}\left(x+t^{2}\right)\right)+V\left(x\left(3 x+2 t^{2}\right), 3 y^{2}-x^{3}-t^{2} x^{2}\right)= \\
2 V(x, y)+V\left(x+t^{2}, y\right)+2 V(x, y)+V\left(3 x+2 t^{2}, 3 y^{2}-x^{3}-t^{2} x^{2}\right) .
\end{gathered}
$$

Thus, $\Gamma_{f, t}^{1}=V\left(3 x+2 t^{2}, 3 y^{2}-x^{3}-t^{2} x^{2}\right)$, and $\left(\Gamma_{f, t}^{1} \cdot V(t)\right)_{\mathbf{0}}=\left[V\left(3 x+2 t^{2}, 3 y^{2}-x^{3}-t^{2} x^{2}, t\right)\right]_{\mathbf{0}}=2$, and $H^{k-0}\left(\mathbb{N}_{\{\mathbf{0}\}}, \mathbb{L}_{\{\mathbf{0}\}} ; \mathbf{F}^{\bullet}\right)=H^{k+2}\left(\mathbb{N}_{\{\mathbf{0}\}}, \mathbb{L}_{\{\mathbf{0}\}} ; \mathbb{Z}\right)$ is isomorphic to $\mathbb{Z}^{2}$ if $k=0$, and is 0 if $k \neq 0$.

Therefore, we find that $\operatorname{gecc}^{k}\left(\mathbf{F}^{\bullet}\right)=0$ if $k \neq 0$, and

$$
\operatorname{gecc}^{0}\left(\mathbf{F}^{\bullet}\right)=\mathbb{Z}\left[\overline{T_{S_{1}}^{*} \mathbb{C}^{3}}\right]+\mathbb{Z}\left[\overline{T_{S_{2}}^{*} \mathbb{C}^{3}}\right]+\mathbb{Z}^{2}\left[\overline{T_{S_{3}}^{*} \mathbb{C}^{3}}\right]+\mathbb{Z}\left[\overline{T_{S_{4}}^{*} \mathbb{C}^{3}}\right]+\mathbb{Z}^{2}\left[T_{\{\mathbf{0}\}}^{*} \mathbb{C}^{3}\right] .
$$

The fact that $\operatorname{gecc}^{\bullet}\left(\mathbf{F}^{\bullet}\right)$ is concentrated in degree 0 is equivalent to the fact that $\mathbb{Z}_{X}^{\bullet}[2]$ is a perverse sheaf (see [17), and was the reason for including the shift by 2 . The constant sheaf on any connected, local complete intersection, shifted by the dimension of the space, is perverse.

The reader is invited to take the most simple space $Y$ which is not a local complete intersection - two planes $P_{1}$ and $P_{2}$ in $\mathbb{C}^{4}$, which intersect at only the origin - and show that, if $\mathbf{A}^{\bullet}=\mathbb{Z}_{Y}^{\bullet}[2]$, then

$$
\begin{gathered}
\operatorname{gecc}^{0}\left(\mathbf{A}^{\bullet}\right)=\mathbb{Z}\left[T_{P_{1}}^{*} \mathbb{C}^{4}\right]+\mathbb{Z}\left[T_{P_{2}}^{*} \mathbb{C}^{4}\right] \\
\operatorname{gecc}^{-1}\left(\mathbf{A}^{\bullet}\right)=\mathbb{Z}\left[T_{\{\mathbf{0}\}}^{*} \mathbb{C}^{4}\right]
\end{gathered}
$$

and $\operatorname{gecc}^{k}\left(\mathbf{A}^{\bullet}\right)=0$ for $k \neq 0,-1$.

\section{The Main Definitions and Theorem}

Throughout the remainder of this paper, we will use the notation established in Section $\mathbb{U} \mathcal{U}$ is an open neighborhood of the origin in $\mathbb{C}^{n+1}, \tilde{f}$ and $\tilde{g}$ are analytic functions from $(\mathcal{U}, \mathbf{0})$ to $(\mathbb{C}, 0), X$ is a complex analytic subset of $\mathcal{U}, f$ and $g$ denote the restrictions of $\tilde{f}$ and $\tilde{g}$, respectively, to $X$, and $\mathfrak{S}$ is a Whitney stratification of $X$, with connected strata, such that $V(f)$ is a union of strata.

We use $\left(z_{0}, \ldots, z_{n}\right)$ for coordinates on $\mathcal{U}$, and identify $T^{*} \mathcal{U}$ with $\mathcal{U} \times \mathbb{C}^{n+1}$, using $\left(w_{0}, \ldots, w_{n}\right)$ for cotangent coordinates, so that $\left(p, w_{0} d_{p} z_{0}+\cdots+w_{n} d_{p} z_{n}\right)$ corresponds to $\left(p,\left(w_{0}, \ldots, w_{n}\right)\right)$. Let $\pi: T^{*} \mathcal{U} \rightarrow \mathcal{U}$ denote the projection. Below, we consider the image, im $d \tilde{g}$, of $d \tilde{g}$ in $T^{*} \mathcal{U}$; this scheme is defined by

$$
V\left(w_{0}-\frac{\partial \tilde{g}}{\partial z_{0}}, \ldots, w_{n}-\frac{\partial \tilde{g}}{\partial z_{n}}\right) \subseteq \mathcal{U} \times \mathbb{C}^{n+1} .
$$

We will consider im $d \tilde{g}$ as a scheme, an analytic set, an ordinary cycle, and as a graded, enriched cycle; we will denote all of these by simply im $d \tilde{g}$, and explicitly state what structure we are using or let the context make the structure clear.

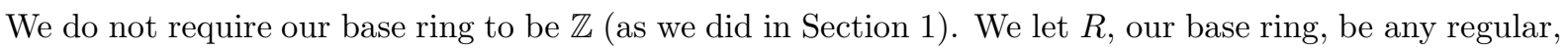
Noetherian ring with finite Krull dimension (e.g., $\mathbb{Z}, \mathbb{Q}$, or $\mathbb{C}$ ). This implies that every finitely-generated $R$-module has finite projective dimension (in fact, it implies that the projective dimension of the module 
is at most $\operatorname{dim} R$ ). We let $\mathbf{F}^{\bullet}$ be a bounded, constructible complex of sheaves of $R$-modules on $X$. Let $\mathfrak{S}\left(\mathbf{F}^{\bullet}\right):=\left\{S \in \mathfrak{S} \mid \mathbb{H}^{*}\left(\mathbb{N}_{S}, \mathbb{L}_{S} ; \mathbf{F}^{\bullet}\right) \neq 0\right\}$; we refer to the elements of $\mathfrak{S}\left(\mathbf{F}^{\bullet}\right)$ as the $\mathbf{F}^{\bullet}$-visible strata of $\mathfrak{S}$.

Suppose that $M$ is a complex submanifold of $\mathcal{U}$. Recall:

Definition 3.1. The relative conormal space $T_{\tilde{f}_{\left.\right|_{M}}}^{*} \mathcal{U}$ is given by

$$
T_{\tilde{f}_{M}}^{*} \mathcal{U}:=\left\{(x, \eta) \in T^{*} \mathcal{U} \mid \eta\left(T_{x} M \cap \operatorname{ker} d_{x} \tilde{f}\right)=0\right\} .
$$

If $M \subseteq X$, then $T_{\tilde{f}_{\mid M}}^{*} \mathcal{U}$ depends on $f$, but not on the particular extension $\tilde{f}$. In this case, we write $T_{f_{\left.\right|_{M}}}^{*} \mathcal{U}$ in place of $T_{\tilde{f}_{\mid M}}^{*} \mathcal{U}$.

Definition 3.2. The graded, enriched relative conormal cycle, $\left(T_{f, \mathrm{~F}}^{*} \mathcal{U}\right)^{\bullet}$, of $f$, with respect to $\mathbf{F}^{\bullet}$, is defined by

$$
\left(T_{f, \mathbf{F}}^{*} \mathcal{U}\right)^{k}:=\sum_{\substack{S \in \mathfrak{S}_{\left(\mathbf{F}^{\bullet}\right)} \\ f_{\left.\right|_{S}} \neq \text { const. }}} H^{k-d_{S}}\left(\mathbb{N}_{S}, \mathbb{L}_{S} ; \mathbf{F}^{\bullet}\right)\left[\overline{T_{f_{\mid S}}^{*} \mathcal{U}}\right]
$$

Example 3.3. Let us return to the setting of Example 2.6 where $X=V(y) \cup V\left(y^{2}-x^{3}-t^{2} x^{2}\right)$ and $\mathbf{F}^{\bullet}=$ $\mathbb{Z}_{X}^{\bullet}[2]$. We had Whitney strata consisting of $\{\mathbf{0}\}, S_{1}=V(y)-V\left(y^{2}-x^{3}-t^{2} x^{2}\right), S_{2}=V\left(y^{2}-x^{3}-t^{2} x^{2}\right)-V(y)$, $S_{3}=V(x, y)-\{\mathbf{0}\}$, and $S_{4}=V\left(x+t^{2}, y\right)-\{\mathbf{0}\}$.

We found that $\operatorname{gecc}^{k}\left(\mathbf{F}^{\bullet}\right)=0$ if $k \neq 0$, and

$$
\operatorname{gecc}^{0}\left(\mathbf{F}^{\bullet}\right)=\mathbb{Z}\left[\overline{T_{S_{1}}^{*} \mathbb{C}^{3}}\right]+\mathbb{Z}\left[\overline{T_{S_{2}}^{*} \mathbb{C}^{3}}\right]+\mathbb{Z}^{2}\left[\overline{T_{S_{3}}^{*} \mathbb{C}^{3}}\right]+\mathbb{Z}\left[\overline{T_{S_{4}}^{*} \mathbb{C}^{3}}\right]+\mathbb{Z}^{2}\left[T_{\{\mathbf{0}\}}^{*} \mathbb{C}^{3}\right] .
$$

We will calculate $\left(T_{x, \mathbf{F} \bullet}^{*} \mathbb{C}^{3}\right)^{\bullet}$.

As we said above, we identify $T^{*} \mathbb{C}^{3}$ with $\mathbb{C}^{3} \times \mathbb{C}^{3}$, and will use coordinates $\left(w_{0}, w_{1}, w_{2}\right)$ for cotangent coordinates, so that $\left(w_{0}, w_{1}, w_{2}\right)$ represents $w_{0} d x+w_{1} d y+w_{2} d t$.

Since $x$ is identically zero on $\{\mathbf{0}\}$ and $S_{3}$, these two strata are not used in the calculation of $\left(T_{x, \mathbf{F}}^{*} \cdot \mathbb{C}^{3}\right)^{\bullet}$. For the 1-dimensional stratum $S_{4},\left[\overline{T_{x_{\mid S_{4}}^{*}}^{*} \mathbb{C}^{3}}\right]$ is the 4-dimensional cycle $V\left(x+t^{2}, y\right) \subseteq \mathbb{C}^{3} \times \mathbb{C}^{3}$.

The fiber of $T_{x_{\mid S_{1}}}^{*} \mathbb{C}^{3}$ over any $p \in S_{1}$ is

$$
\left(T_{S_{1}}^{*} \mathbb{C}^{3}\right)_{p}+<d_{p} x>:=\left\{\omega+a d_{p} x \mid \omega \in\left(T_{S_{1}}^{*} \mathbb{C}^{3}\right)_{p}, a \in \mathbb{C}\right\}=\left\{b d_{p} y+a d_{p} x \mid a, b \in \mathbb{C}\right\} .
$$

Hence, $\left[\overline{T_{x_{S_{1}}}^{*} \mathbb{C}^{3}}\right]=V\left(y, w_{2}\right)$.

The fiber of $T_{x_{S_{2}}}^{*} \mathbb{C}^{3}$ over any $p \in S_{2}$ which is a regular point of $x$ restricted to $S_{2}$ is

$$
\begin{gathered}
\left(T_{S_{2}}^{*} \mathbb{C}^{3}\right)_{p}+<d_{p} x>:= \\
\left\{\omega+a d_{p} x \mid \omega \in\left(T_{S_{2}}^{*} \mathbb{C}^{3}\right)_{p}, a \in \mathbb{C}\right\}=\left\{b\left(\left(-3 x^{2}-2 t^{2} x\right) d_{p} x+2 y d_{p} y-2 t x^{2} d_{p} t\right)+a d_{p} x \mid a, b \in \mathbb{C}\right\} .
\end{gathered}
$$

The form $w_{0} d_{p} x+w_{1} d_{p} y+w_{2} d_{p} t$ is in this set if and only if the determinant of the following matrix is 0 :

$$
\left[\begin{array}{ccc}
w_{0} & w_{1} & w_{2} \\
-3 x^{2}-2 t^{2} x & 2 y & -2 t x^{2} \\
1 & 0 & 0
\end{array}\right]
$$


i.e., if and only if $y w_{2}+t x^{2} w_{1}=0$. It is tempting to conclude that $\left[\overline{T_{x_{S_{2}}^{*}}^{*} \mathbb{C}^{3}}\right]$ equals $V\left(y^{2}-x^{3}-t^{2} x^{2}, y w_{2}+\right.$ $\left.t x^{2} w_{1}\right)$, but this is not the case; we must eliminate any components of $V\left(y^{2}-x^{3}-t^{2} x^{2}, y w_{2}+t x^{2} w_{1}\right)$ which are contained in $V(x, y)$. Our notation for the resulting scheme (a gap sheaf, see [16], I.1) is

$$
V\left(y^{2}-x^{3}-t^{2} x^{2}, y w_{2}+t x^{2} w_{1}\right) \neg V(x, y) .
$$

Note that, as schemes,

$$
\begin{gathered}
V\left(y^{2}-x^{3}-t^{2} x^{2}, y w_{2}+t x^{2} w_{1}\right)=V\left(y^{2}-x^{3}-t^{2} x^{2}, y w_{2}+t x^{2} w_{1}, y^{2} w_{2}+y t x^{2} w_{1}\right)= \\
V\left(y^{2}-x^{3}-t^{2} x^{2}, y w_{2}+t x^{2} w_{1},\left(x^{3}+t^{2} x^{2}\right) w_{2}+y t x^{2} w_{1}\right) .
\end{gathered}
$$

Using [16, I.1.3.iv, we find that, as cycles,

$$
V\left(y^{2}-x^{3}-t^{2} x^{2}, y w_{2}+t x^{2} w_{1}\right) \neg V(x, y)=V\left(y^{2}-x^{3}-t^{2} x^{2}, y w_{2}+t x^{2} w_{1},\left(x+t^{2}\right) w_{2}+y t w_{1}\right) .
$$

(This last equality need not be true on the level of schemes, since our generators do not form a regular sequence and, hence, there may be embedded subvarieties.)

Therefore, we find that $\left(T_{x, \mathbf{F}}^{*} \mathbb{C}^{3}\right)^{k}$ is 0 unless $k=0$, and

$$
\left(T_{x, \mathbf{F} \bullet}^{*} \mathbb{C}^{3}\right)^{0}=\mathbb{Z}\left[V\left(y, w_{2}\right)\right]+\mathbb{Z}\left[V\left(y^{2}-x^{3}-t^{2} x^{2}, y w_{2}+t x^{2} w_{1},\left(x+t^{2}\right) w_{2}+y t w_{1}\right)\right]+\mathbb{Z}\left[V\left(x+t^{2}, y\right)\right] .
$$

We now wish to define the graded, enriched relative polar curve. Note that the projection $\pi$ induces an isomorphism from the analytic set $\operatorname{im} d \tilde{g}$ to $\mathcal{U}$. We will use the proper push-forward (Definition 2.2) of the map $\pi$ restricted to im $d \tilde{g}$; we will continue to denote this restriction by simply $\pi$.

By our conventions in Section 2 the graded, enriched im $d \tilde{g}$ is zero outside of degree 0 , and is $R[\mathrm{im} d \tilde{g}]$ in degree 0.

Definition 3.4. If $S \in \mathfrak{S}$ and $f_{\left.\right|_{S}}$ is not constant, we define the relative polar set, $\left|\Gamma_{f, \tilde{g}}(S)\right|$, to be $\pi\left(\overline{T_{f_{\mid S}}^{*} \mathcal{U}} \cap \operatorname{im} d \tilde{g}\right)$; if this set is purely 1-dimensional, so that $\overline{T_{f_{\mid S}}^{*} \mathcal{U}}$ and im $d \tilde{g}$ intersect properly, we define the (ordinary) relative polar cycle, $\Gamma_{f, \tilde{g}}(S)$, to be the cycle $\pi_{*}\left(\left[\overline{T_{f_{\mid S}^{*}}^{*} \mathcal{U}}\right] \cdot[\operatorname{im} d \tilde{g}]\right)$.

The relative polar set, $\left|\Gamma_{f, \tilde{g}}\left(\mathbf{F}^{\bullet}\right)\right|$, is defined by

$$
\left|\Gamma_{f, \tilde{g}}\left(\mathbf{F}^{\bullet}\right)\right|:=\pi\left(\left|\left(T_{f, \mathbf{F}}^{*} \cdot \mathcal{U}\right)^{\bullet}\right| \cap \operatorname{im} d \tilde{g}\right) .
$$

Each 1-dimensional component $C$ of $\left|\Gamma_{f, \tilde{g}}\left(\mathbf{F}^{\bullet}\right)\right|$ is the image of a component of $\left|\left(T_{f, \mathbf{F}}^{*} \mathcal{U}\right)^{\bullet}\right| \cap \operatorname{im} d \tilde{g}$ along which $\left|\left(T_{f, \mathbf{F} \cdot}^{*} \mathcal{U}\right)^{\bullet}\right|$ and im $d \tilde{g}$ intersect properly. We give such a component $C$ the structure of the graded, enriched cycle whose underlying set is $C$ and whose graded, enriched cycle structure is given by $\pi_{*}^{\bullet}\left(\left(T_{f, \mathbf{F} \cdot}^{*} \mathcal{U}\right)^{\bullet} \odot \operatorname{im} d \tilde{g}\right)$ over generic points in $C$. We refer to this as the graded, enriched cycle structure of $C$ in $\left|\Gamma_{f, \tilde{g}}\left(\mathbf{F}^{\bullet}\right)\right|$.

If $\left|\Gamma_{f, \tilde{g}}\left(\mathbf{F}^{\bullet}\right)\right|$ is purely 1-dimensional, we say that the graded, enriched relative polar curve, $\left(\Gamma_{f, \tilde{g}}^{1}\left(\mathbf{F}^{\bullet}\right)\right)^{\bullet}$, is defined, and is given by

$$
\left(\Gamma_{f, \tilde{g}}^{1}\left(\mathbf{F}^{\bullet}\right)\right)^{\bullet}:=\pi_{*}^{\bullet}\left(\left(T_{f, \mathbf{F}}^{*} \mathcal{U}\right)^{\bullet} \odot \operatorname{im} d \tilde{g}\right),
$$


i.e.,

$$
\left(\Gamma_{f, \tilde{g}}^{1}\left(\mathbf{F}^{\bullet}\right)\right)^{k}=\sum_{\substack{S \in \mathfrak{S}\left(\mathbf{F}^{\bullet}\right) \\ f_{\mid S} \neq \text { const. }}} H^{k-d_{S}}\left(\mathbb{N}_{S}, \mathbb{L}_{S} ; \mathbf{F}^{\bullet}\right)\left(\Gamma_{f, \tilde{g}}(S)\right)^{\mathrm{enr}}
$$

Remark 3.5. In the notation for the polar curve, we write $\tilde{g}$, not simply $g$; we do not, in fact, know if $\left(\Gamma_{f, \tilde{g}}^{1}\left(\mathbf{F}^{\bullet}\right)\right)^{\bullet}$ is independent of the extension to $\tilde{g}$. However, Theorem 3.11 will imply that, when $\left(\Gamma_{f, \tilde{g}}^{1}\left(\mathbf{F}^{\bullet}\right)\right)^{\bullet}$ is defined and has no component on which $f$ is constant, then $\left(\Gamma_{f, \tilde{g}}^{1}\left(\mathbf{F}^{\bullet}\right)\right)^{\bullet}$ is independent of the extension $\tilde{g}$. It is also not difficult to show that the set $\left|\Gamma_{f, \tilde{g}}\left(\mathbf{F}^{\bullet}\right)\right|$ is independent of the extension of $g$, but we shall not need this result here.

Note that $\overline{T_{f_{\mid S}}^{*} \mathcal{U}} \cap \operatorname{im} d \tilde{g}$ is at least 1-dimensional at each point of intersection, and so $\left|\Gamma_{f, \tilde{g}}\left(\mathbf{F}^{\bullet}\right)\right|$ has no isolated points. Also, note that, as $\left|\left(T_{f, \mathbf{F} \bullet}^{*} \mathcal{U}\right)^{\bullet}\right| \cap \operatorname{im} d \tilde{g}$ is a closed subset of im $d \tilde{g}$, and $\pi$ induces an isomorphism from im $d \tilde{g}$ to $\mathcal{U},\left|\Gamma_{f, \tilde{g}}\left(\mathbf{F}^{\bullet}\right)\right|$ is a closed subset of $\mathcal{U}$.

Finally, the reader may wonder about the symmetry of our definition. It is not true for arbitrary $\tilde{f}$ and $\tilde{g}$ that even the sets $\left|\Gamma_{f, \tilde{g}}\left(\mathbf{F}^{\bullet}\right)\right|$ and $\left|\Gamma_{g, \tilde{f}}\left(\mathbf{F}^{\bullet}\right)\right|$ are equal; see Remark 4.11] However, Proposition 4.10 will imply that the components of these two sets along which neither $f$ nor $g$ are constant are the same. Hence, we refer to a component of $\left|\Gamma_{f, \tilde{g}}\left(\mathbf{F}^{\bullet}\right)\right|$ along which neither $f$ nor $g$ is is constant as a symmetric component of $\left|\Gamma_{f, \tilde{g}}\left(\mathbf{F}^{\bullet}\right)\right|$.

By moving to a generic point $p$ on a 1-dimensional symmetric component $C$ of $\left|\Gamma_{f, \tilde{g}}\left(\mathbf{F}^{\bullet}\right)\right|$ and applying Theorem 4.13, one can show that the graded, enriched cycle structure of $C$ in $\left|\Gamma_{f, \tilde{g}}\left(\mathbf{F}^{\bullet}\right)\right|$ is the same as that of $C$ in $\left|\Gamma_{g, \tilde{f}}\left(\mathbf{F}^{\bullet}\right)\right|$.

Example 3.6. We continue with our setting from Example 2.6 and Example 3.3 and consider $X=$ $V(y) \cup V\left(y^{2}-x^{3}-t^{2} x^{2}\right)$ and $\mathbf{F}^{\bullet}=\mathbb{Z}_{X}^{\bullet}[2]$. We will calculate $\left(\Gamma_{x, t}^{1}\left(\mathbf{F}^{\bullet}\right)\right)^{\bullet}$.

Using the isomorphism $T^{*} \mathbb{C}^{3} \cong \mathbb{C}^{3} \times \mathbb{C}^{3}$ from Example 3.3 im $d t$ is the scheme

$$
V\left(w_{0}-\frac{\partial t}{\partial x}, w_{1}-\frac{\partial t}{\partial y}, w_{2}-\frac{\partial t}{\partial t}\right)=V\left(w_{0}, w_{1}, w_{2}-1\right)
$$

In Example 3.3 we found that $\left(T_{x, \mathbf{F}}^{*} \cdot \mathbb{C}^{3}\right)^{k}$ is 0 unless $k=0$, and

$$
\left(T_{x, \mathbf{F} \bullet}^{*} \mathbb{C}^{3}\right)^{0}=\mathbb{Z}\left[V\left(y, w_{2}\right)\right]+\mathbb{Z}\left[V\left(y^{2}-x^{3}-t^{2} x^{2}, y w_{2}+t x^{2} w_{1},\left(x+t^{2}\right) w_{2}+y t w_{1}\right)\right]+\mathbb{Z}\left[V\left(x+t^{2}, y\right)\right] .
$$

Let us write $E$ for the cycle $V\left(y^{2}-x^{3}-t^{2} x^{2}, y w_{2}+t x^{2} w_{1},\left(x+t^{2}\right) w_{2}+y t w_{1}\right)$ throughout the remainder of this example.

Thus, $\left(\Gamma_{x, t}^{1}\left(\mathbf{F}^{\bullet}\right)\right)^{k}$ is 0 unless $k=0$ and, to calculate $\left(\Gamma_{x, t}^{1}\left(\mathbf{F}^{\bullet}\right)\right)^{0}$, we need first to calculate the three ordinary cycles

$$
\begin{gathered}
\pi_{*}\left(V\left(y, w_{2}\right) \cdot V\left(w_{0}, w_{1}, w_{2}-1\right)\right), \\
\pi_{*}\left(E \cdot V\left(w_{0}, w_{1}, w_{2}-1\right)\right),
\end{gathered}
$$

and

$$
\pi_{*}\left(V\left(x+t^{2}, y\right) \cdot V\left(w_{0}, w_{1}, w_{2}-1\right)\right) .
$$

Now, $V\left(y, w_{2}\right) \cap V\left(w_{0}, w_{1}, w_{2}-1\right)=\emptyset$, and so $\pi_{*}\left(V\left(y, w_{2}\right) \cdot V\left(w_{0}, w_{1}, w_{2}-1\right)\right)=0$. In addition, it is trivial that there is an equality of cycles $\pi_{*}\left(V\left(x+t^{2}, y\right) \cdot V\left(w_{0}, w_{1}, w_{2}-1\right)\right)=V\left(x+t^{2}, y\right)$. However, the remaining cycle is more difficult to calculate. 
The difficulty in calculating

$$
\pi_{*}\left(E \cdot V\left(w_{0}, w_{1}, w_{2}-1\right)\right)
$$

is due to the fact that $y^{2}-x^{3}-t^{2} x^{2}, y w_{2}+t x^{2} w_{1},\left(x+t^{2}\right) w_{2}+y t w_{1}$ is not a regular sequence. To "fix" this, note that, in Example 3.3 we saw that, as cycles, there is an equality

$$
V\left(y^{2}-x^{3}-t^{2} x^{2}, y w_{2}+t x^{2} w_{1}\right)=C+E,
$$

where the underlying set $|C| \subseteq V(x, y)$. Now, it is trivial that, as sets,

$$
E \cap V\left(w_{0}, w_{1}, w_{2}-1\right)=V\left(x+t^{2}, y, w_{0}, w_{1}, w_{2}-1\right) .
$$

Therefore,

$$
\begin{gathered}
C \cdot V\left(w_{0}, w_{1}, w_{2}-1\right)+E \cdot V\left(w_{0}, w_{1}, w_{2}-1\right)=V\left(y^{2}-x^{3}-t^{2} x^{2}, y w_{2}+t x^{2} w_{1}\right) \cdot V\left(w_{0}, w_{1}, w_{2}-1\right)= \\
V\left(y^{2}-x^{3}-t^{2} x^{2}, y w_{2}+t x^{2} w_{1}, w_{0}, w_{1}, w_{2}-1\right)=V\left(x^{2}\left(x+t^{2}\right), y, w_{0}, w_{1}, w_{2}-1\right) \\
2 V\left(x, y, w_{0}, w_{1}, w_{2}-1\right)+V\left(x+t^{2}, y, w_{0}, w_{1}, w_{2}-1\right) .
\end{gathered}
$$

Thus, as cycles,

$$
E \cdot V\left(w_{0}, w_{1}, w_{2}-1\right)=V\left(x+t^{2}, y, w_{0}, w_{1}, w_{2}-1\right),
$$

and so $\pi_{*}\left(E \cdot V\left(w_{0}, w_{1}, w_{2}-1\right)\right)=V\left(x+t^{2}, y\right)$.

Finally, we find that

$$
\left(\Gamma_{x, t}^{1}\left(\mathbf{F}^{\bullet}\right)\right)^{0}=\pi_{*}^{0}\left(\left(T_{f, \mathbf{F}}^{*} \mathcal{U}\right) \bullet \operatorname{im} d t\right)=\mathbb{Z}\left[V\left(x+t^{2}, y\right)\right]+\mathbb{Z}\left[V\left(x+t^{2}, y\right)\right]=\mathbb{Z}^{2}\left[V\left(x+t^{2}, y\right)\right] .
$$

Before we can prove our main theorem of this section, we must recall three results from [17].

Theorem 3.7. (17, Theorem 3.3) There is an equality of graded enriched cycles given by

$$
\operatorname{gecc}^{\bullet}\left(\psi_{f}[-1] \mathbf{F}^{\bullet}\right)=\left(T_{f, \mathbf{F} \bullet}^{*} \mathcal{U}\right)^{\bullet} \odot\left(V(f) \times \mathbb{C}^{n+1}\right) .
$$

We state the next two theorems for complexes of sheaves on $V(f)$, since that is the case in which we shall use them.

Theorem 3.8.(17], Theorem 3.4) Let $\mathbf{A}^{\bullet}$ be a bounded, constructible complex of sheaves of $R$-modules on $V(f)$. Then, $\operatorname{dim}_{\mathbf{0}}\left(\operatorname{supp} \phi_{g}[-1] \mathbf{A}^{\bullet}\right) \leq 0$ if and only if $\operatorname{dim}_{\mathbf{0}} \pi\left(\left|\operatorname{gecc}^{\bullet}\left(\mathbf{A}^{\bullet}\right)\right| \cap \operatorname{im} d \tilde{g}\right) \leq 0$, and when this is the case, $\operatorname{dim}_{\left(\mathbf{0}, d_{0} \tilde{g}\right)}\left(\left|\operatorname{gecc} \bullet\left(\mathbf{A}^{\bullet}\right)\right| \cap \operatorname{im} d \tilde{g}\right) \leq 0$ and

$$
H^{k}\left(\phi_{g}[-1] \mathbf{A}^{\bullet}\right)_{\mathbf{0}} \cong\left(\operatorname{gecc}^{k}\left(\mathbf{A}^{\bullet}\right) \odot \operatorname{imd} d \tilde{g}\right)_{\left(\mathbf{0}, d_{\mathbf{o}} \tilde{g}\right)} .
$$

Theorem 3.9. (17, Theorem 3.5) Let $\mathbf{A}^{\bullet}$ be a bounded, constructible complex of sheaves of $R$-modules on $V(f)$. Then, there is an equality of sets given by

$$
\bigcup_{v \in \mathbb{C}} \operatorname{supp} \phi_{g-v}[-1] \mathbf{A}^{\bullet}=\pi\left(\left|\operatorname{gecc}{ }^{\bullet}\left(\mathbf{A}^{\bullet}\right)\right| \cap \operatorname{im} d \tilde{g}\right) .
$$


We need a lemma before we prove our first main theorem.

Lemma 3.10. There is an equality of sets

$$
\operatorname{supp} \phi_{g}[-1] \psi_{f}[-1] \mathbf{F}^{\bullet}=V(f, g) \cap\left|\Gamma_{f, \tilde{g}}\left(\mathbf{F}^{\bullet}\right)\right|,
$$

and, in a neighborhood of the origin, $V(f) \cap\left|\Gamma_{f, \tilde{g}}\left(\mathbf{F}^{\bullet}\right)\right| \subseteq V(g)$.

Proof. The equality follows from Theorem 3.9] by letting $\mathbf{A}^{\bullet}=\psi_{f}[-1] \mathbf{F}^{\bullet}$, applying Theorem 3.7 and then intersecting $V(g)$ with both sides of the equation from Theorem 3.9. The containment also follows from Theorem 3.9] by letting $\mathbf{A}^{\bullet}=\psi_{f}[-1] \mathbf{F}^{\bullet}$, applying Theorem 3.7 and then using that, near a point $p$ where $g(p)=0, \operatorname{supp} \phi_{g-v}[-1] \mathbf{A}^{\bullet}$ is empty if $v \neq 0$.

We now prove our first main theorem.

Theorem 3.11. The following are equivalent:

1. $\operatorname{dim}_{\mathbf{0}} \operatorname{supp} \phi_{g}[-1] \psi_{f}[-1] \mathbf{F}^{\bullet} \leq 0$;

2. $\operatorname{dim}_{0} V(f) \cap\left|\Gamma_{f, \tilde{g}}\left(\mathbf{F}^{\bullet}\right)\right| \leq 0$;

3. $\operatorname{dim}_{0} V(f, g) \cap\left|\Gamma_{f, \tilde{g}}\left(\mathbf{F}^{\bullet}\right)\right| \leq 0$;

and, when these equivalent conditions hold, $\left(\Gamma_{f, \tilde{g}}^{1}\left(\mathbf{F}^{\bullet}\right)\right)^{\bullet}$ exists and

$$
H^{k}\left(\phi_{g}[-1] \psi_{f}[-1] \mathbf{F}^{\bullet}\right)_{\mathbf{0}} \cong\left(\left(\Gamma_{f, \tilde{g}}^{1}\left(\mathbf{F}^{\bullet}\right)\right)^{k} \odot V(f)\right)_{\mathbf{0}},
$$

i.e.,

$$
H^{k-1}\left(B_{\epsilon} \cap f^{-1}(\xi), B_{\epsilon} \cap f^{-1}(\xi) \cap g^{-1}(\nu) ; \mathbf{F}^{\bullet}\right) \cong \bigoplus_{S \in \mathfrak{S}^{\circ}\left(\mathbf{F}^{\bullet}\right)}\left(\mathbb{H}^{k-d_{S}}\left(\mathbb{N}_{S}, \mathbb{L}_{S} ; \mathbf{F}^{\bullet}\right)\right)^{j_{S}},
$$

where $0<|\xi| \ll|\nu|<\ll \epsilon \ll 1$, and $j_{S}=\left(\Gamma_{f, \tilde{g}}^{1}(S) \cdot V(f)\right)_{\mathbf{0}}$.

Proof. The equivalence of the conditions follows immediately from the lemma. Assume now that these conditions hold.

By Theorem 3.8

$$
H^{k}\left(\phi_{g}[-1] \psi_{f}[-1] \mathbf{F}^{\bullet}\right)_{\mathbf{0}} \cong\left(\operatorname{gecc}^{k}\left(\psi_{f}[-1] \mathbf{F}^{\bullet}\right) \odot \operatorname{im} d \tilde{g}\right)_{\left(\mathbf{0}, d_{\mathbf{0}} \tilde{g}\right)} .
$$

Applying Theorem 3.7 we find that

$$
\begin{gathered}
H^{k}\left(\phi_{g}[-1] \psi_{f}[-1] \mathbf{F}^{\bullet}\right)_{\mathbf{0}} \cong\left(\left(T_{f, \mathbf{F}}^{*} \mathcal{U}\right)^{k} \odot\left(V(f) \times \mathbb{C}^{n+1}\right) \odot \operatorname{im} d \tilde{g}\right)_{\left(\mathbf{0}, d_{\mathbf{0}} \tilde{g}\right)} \cong \\
\left(\pi_{*}^{k}\left(\left(T_{f, \mathbf{F}}^{*} \mathcal{U}\right)^{\bullet} \odot\left(V(f) \times \mathbb{C}^{n+1}\right) \odot \operatorname{im} d \tilde{g}\right)\right)_{\mathbf{0}},
\end{gathered}
$$

where this last isomorphism follows from the definition of the proper push-forward. By Proposition[2.3 this last quantity equals

$$
\left(\pi_{*}^{k}\left(\left(T_{f, \mathbf{F}}^{*} \mathcal{U}\right)^{\bullet} \odot \operatorname{im} d \tilde{g}\right) \odot V(f)\right)_{\mathbf{0}}
$$


which, by definition of the graded, enriched relative polar curve is equal to $\left(\left(\Gamma_{f, \tilde{g}}^{1}\left(\mathbf{F}^{\bullet}\right)\right)^{k} \odot V(f)\right)_{\mathbf{0}}$.

We would like to know, of course, that the equivalent hypotheses of Theorem 3.11 are satisfied in the classical case where $f$ is fixed and $\tilde{g}$ is chosen to be a generic linear form.

\section{Proposition 3.12.}

1. There exists a non-zero linear form $\mathfrak{l}$ such that $\mathbf{0} \notin\left|\Gamma_{f, \mathfrak{l}}\left(\mathbf{F}^{\bullet}\right)\right|$ if and only if for generic linear $\mathfrak{l}$, $\mathbf{0} \notin\left|\Gamma_{f, l}\left(\mathbf{F}^{\bullet}\right)\right|$.

2. For generic linear $\mathfrak{l}, \operatorname{dim}_{\mathbf{0}} V(f) \cap\left|\Gamma_{f, \mathfrak{l}}\left(\mathbf{F}^{\bullet}\right)\right| \leq 0$ and $\operatorname{dim}_{\mathbf{0}} V(\mathfrak{l}) \cap\left|\Gamma_{f, \mathfrak{l}}\left(\mathbf{F}^{\bullet}\right)\right| \leq 0$.

Proof. The proof of Item 1 is standard. Suppose that there exists a non-zero linear form $\mathfrak{l}$ such that $\mathbf{0} \notin$ $\left|\Gamma_{f, \mathfrak{l}}\left(\mathbf{F}^{\bullet}\right)\right|$. Then, the projective class $\left[d_{\mathbf{0}} \mathfrak{l}\right]$ is not in the fiber $\mathbb{P}\left(\left|\left(T_{f, \mathbf{F}}^{*} \mathcal{U}\right)^{\bullet}\right|\right)_{\mathbf{0}} \subseteq \mathbb{P}^{n}$. Thus, $\mathbb{P}\left(\left|\left(T_{f, \mathbf{F}}^{*} \mathcal{U}\right)^{\bullet}\right|\right)_{\mathbf{0}}$ is a proper analytic subset of $\mathbb{P}^{n}$. This implies Item 1.

Proof of Item 2:

If $\mathbf{A}^{\bullet}$ is any bounded, constructible complex of sheaves on any complex analytic $Y \subset \mathcal{U}$ and $\mathbf{0} \in Y$, then for generic linear $\mathfrak{l}$ on $U, \mathbf{0}$ is an isolated point in $\operatorname{supp} \phi_{l}[-1] \mathbf{A}^{\bullet}$. This is well-known; see, for instance, Theorem 2.4 of [15]. Thus, for generic $\mathfrak{l}, \operatorname{dim}_{0} \operatorname{supp} \phi_{\mathfrak{r}}[-1] \psi_{f}[-1] \mathbf{F}^{\bullet} \leq 0$, which, by Theorem 3.11 is equivalent to $\operatorname{dim}_{\mathbf{0}} V(f) \cap\left|\Gamma_{f, l}\left(\mathbf{F}^{\bullet}\right)\right| \leq 0$.

Now, refine, if necessary, our Whitney stratification $\mathfrak{S}$ to obtain a Whitney stratification $\mathfrak{S}^{\prime}$ which also satisfies Thom's $a_{f}$ condition. For generic linear $\mathfrak{l}, V(\mathfrak{l})$ will transversely intersect all of the strata of $\mathfrak{S}^{\prime}$ in a neighborhood of the origin, except possibly at the origin itself. Fix such an $\mathfrak{l}$. We claim that $\operatorname{dim}_{\mathbf{0}} V(\mathfrak{l}) \cap\left|\Gamma_{f, \mathfrak{l}}\left(\mathbf{F}^{\bullet}\right)\right| \leq 0$.

Since $\mathfrak{S}^{\prime}$ is an $a_{f}$ stratification, $\bigcup_{S \in \mathfrak{S}^{\prime}} \overline{T_{f_{\mid S}}^{*} \mathcal{U}}=\bigcup_{S \in \mathfrak{S}^{\prime}} T_{f_{\mid S}^{*}}^{*} \mathcal{U}$. Our choice of $\mathfrak{l}$ implies that, if $p \neq \mathbf{0}$ and $p \in S \in \mathfrak{S}^{\prime}$, then $d_{p} \mathfrak{l} \notin\left(T_{S}^{*} \mathcal{U}\right)_{p}$. Suppose now that we have an analytic path $\alpha(t)$ such that $\alpha(0)=\mathbf{0}$ and, for $t \neq 0, \alpha(t) \neq \mathbf{0}$ and $\alpha(t) \in V(\mathfrak{l}) \cap\left|\Gamma_{f, \mathfrak{l}}\left(\mathbf{F}^{\bullet}\right)\right|$. We wish to arrive at a contradiction.

If $f(\alpha(t))=0$ when $|t|$ is small, then we are finished, since $\operatorname{dim}_{\mathbf{0}} V(f) \cap\left|\Gamma_{f, \mathrm{l}}\left(\mathbf{F}^{\bullet}\right)\right| \leq 0$. So, assume that for $|t|$ small and non-zero, $f(\alpha(t)) \neq 0$.

For $|t|$ small and non-zero, $\alpha(t)$ is contained in a single stratum $S \in \mathfrak{S}^{\prime}$. Near the origin, the $\mathfrak{S}^{\prime}$-stratified critical locus is contained in $V(f)$; hence, by the assumption in the previous paragraph, for $|t|$ small and nonzero, $d_{\alpha(t)} \tilde{f} \notin\left(T_{S}^{*} \mathcal{U}\right)_{\alpha(t)}$. From our definition of $\alpha(t)$, and the discussion two paragraphs above, it follows that, for $|t|$ small and non-zero, $d_{\alpha(t)} \mathfrak{l} \notin\left(T_{S}^{*} \mathcal{U}\right)_{\alpha(t)}, \mathfrak{l}(\alpha(t)) \equiv 0$, and $d_{\alpha(t)} \mathfrak{l} \in\left(T_{f_{\mid S}^{*}}^{*} \mathcal{U}\right)_{\alpha(t)}=\left(T_{S}^{*} \mathcal{U}\right)_{\alpha(t)}+<d_{\alpha(t)} \tilde{f}>$, where the last equality uses that $d_{\alpha(t)} \tilde{f} \notin\left(T_{S}^{*} \mathcal{U}\right)_{\alpha(t)}$.

Thus, for $|t|$ small and non-zero, there exists $c_{t} \in \mathbb{C}$ such that

$$
d_{\alpha(t)} \mathfrak{l}+c_{t} d_{\alpha(t)} \tilde{f} \in\left(T_{S}^{*} \mathcal{U}\right)_{\alpha(t)}
$$

Evaluating at $\alpha^{\prime}(t)$, and using that $\mathfrak{l}(\alpha(t)) \equiv 0$ and $\alpha^{\prime}(t) \in T_{\alpha(t)} S$, we immediately conclude that $c_{t}(f(\alpha(t)))^{\prime} \equiv 0$. However, $c_{t}$ cannot be zero, for otherwise $(\dagger)$ would imply that $d_{\alpha(t)} \mathfrak{l} \in\left(T_{S}^{*} \mathcal{U}\right)_{\alpha(t)}$. Therefore, we must have that $(f(\alpha(t)))^{\prime} \equiv 0$, which implies that $f(\alpha(t)) \equiv 0$, since $f(\alpha(0))=0$. This is a contradiction. 
Example 3.13. We continue where we left off in Example 3.6 $X=V(y) \cup V\left(y^{2}-x^{3}-t^{2} x^{2}\right)$ and $\mathbf{F}^{\bullet}=\mathbb{Z}_{X}^{\bullet}[2]$. We found that $\left(\Gamma_{x, t}^{1}\left(\mathbf{F}^{\bullet}\right)\right)^{\bullet}$ was concentrated in degree 0 , and

$$
\left(\Gamma_{x, t}^{1}\left(\mathbf{F}^{\bullet}\right)\right)^{0}=\mathbb{Z}^{2}\left[V\left(x+t^{2}, y\right)\right] .
$$

Thus, Theorem 3.11 tells us that $H^{k}\left(\phi_{t}[-1] \psi_{x}[-1] \mathbb{Z}_{X}^{\bullet}[2]\right)_{0}$ is 0 unless $k=0$, and

$$
H^{0}\left(\phi_{t}[-1] \psi_{x}[-1] \mathbb{Z}_{X}^{\bullet}[2]\right)_{\mathbf{0}} \cong\left(\mathbb{Z}^{2}\left[V\left(x+t^{2}, y\right)\right] \odot V(x)\right)_{\mathbf{0}}=\mathbb{Z}^{4} .
$$

\section{The Discriminant as a Complex of Sheaves}

Theorem 3.11] and its elegant, formal proof, was our motivation for defining the graded, enriched relative polar curve as we did. Of course, it would be nice to have a generalization of the result of Lê in its original

form, as it appears in Theorem 1.5 a result which gives $\mathbb{H}^{*}\left(F_{f, \mathbf{0}}, F_{f_{\mid(g)}}, \mathbf{0} ; \mathbf{F}^{\bullet}\right)$. In fact, we could easily prove such a result by appealing to the discriminant and Cerf diagram, if only we could push the complex $\mathbf{F}^{\bullet}$ down to the discriminant in some nice way.

There is one serious technical issue involved: we must show that a suitable neighborhood of origin pushes down by $(g, f)$ to a complex analytically constructible complex, a complex which is constructible with respect to a stratification which is essentially determined by the image of the enriched relative polar curve. The main problem is that, on an open neighborhood of the origin, $(g, f)$ will not be a proper map and, if we instead use a domain with boundary on which $(g, f)$ is proper, then the boundary causes us to leave the complex analytic setting.

This is precisely the type of problem that is addressed by the microlocal theory of Kashiwara and Schapira in [6], and we will use the micro-support of complexes of sheaves on real semianalytic sets. It will take a fair amount of preliminary work before we arrive at the desired result.

Suppose that $M$ is a $C^{\infty}$ manifold, $Z$ is a subspace of $M$, and $\mathbf{A}^{\bullet}$ is a bounded complex of sheaves of $R$-modules on $Z$. Then, Kashiwara and Schapira define the micro-support, $S S\left(\mathbf{A}^{\bullet}\right) \subseteq T^{*} M$, of $\mathbf{A}^{\bullet}$ in 5.1 .2 of [6]. Intuitively, $(p, \eta) \in S S\left(\mathbf{A}^{\bullet}\right)$ if and only if the local hypercohomology of $Z$, with coefficients in $\mathbf{A}^{\bullet}$, changes as one "moves" in the direction of $\eta$.

In our fixed complex analytic setting, where $X$ is a complex analytic subset of $\mathcal{U}$ and $\mathbf{F}^{\bullet}$ is complex analytically constructible, the micro-support is easy to describe.

Proposition 4.1. (14, Theorem 4.13)

$$
S S\left(\mathbf{F}^{\bullet}\right)=\left|\operatorname{gecc}^{\bullet}\left(\mathbf{F}^{\bullet}\right)\right|=\bigcup_{S \in \mathfrak{S}\left(\mathbf{F}^{\bullet}\right)} \overline{T_{S}^{*} \mathcal{U}}
$$

We need to define the critical locus of complex analytic maps relative to the complex $\mathbf{F}^{\bullet}$. If $p \in X$, we shall write $S S_{p}\left(\mathbf{F}^{\bullet}\right)$ for the fiber $\pi^{-1}(p) \cap S S\left(\mathbf{F}^{\bullet}\right)$.

Definition 4.2. The $\mathbf{F}^{\bullet}$ critical locus of $f, \Sigma_{\mathbf{F}} \cdot f$, is the set $\left\{p \in X \mid H^{*}\left(\phi_{f-f(p)}[-1] \mathbf{F}^{\bullet}\right)_{p} \neq 0\right\}$.

Proposition 4.3. (Theorem 2.4 and Remark 2.5 of [15]) The closure $\overline{\Sigma_{\mathbf{F} \bullet f}}$ is equal to $\pi\left(\operatorname{im} d \tilde{f} \cap S S\left(\mathbf{F}^{\bullet}\right)\right)$ and $f$ is constant along the components of $\overline{\Sigma_{\mathbf{F} \bullet f}}$. 
We need to generalize $\overline{\Sigma_{\mathbf{F}} \cdot f}$ to the case where $f$ is a real analytic map whose codomain has dimension greater than one, and where we replace $\mathbf{F}^{\bullet}$ by something more general.

We may consider $T^{*} \mathcal{U}$ with its complex analytic structure, as we have been up to this point, or with its real analytic structure. When it is important for us to distinguish these structures, we will write $\left(T^{*} \mathcal{U}\right)^{\mathbb{C}}$ and $\left(T^{*} \mathcal{U}\right)^{\mathbb{R}}$, respectively, and we remind the reader that, for $p \in \mathcal{U}$, there is an $\mathbb{R}$-linear isomorphism from $\left(T^{*} \mathcal{U}\right)_{p}^{\mathbb{C}}$ to $\left(T^{*} \mathcal{U}\right)_{p}^{\mathbb{R}}$ given by mapping $\eta$ to the real part $\operatorname{Re} \eta($ or $2 \operatorname{Re} \eta)$. If $\eta_{1}, \ldots, \eta_{k} \in\left(T^{*} \mathcal{U}\right)_{p}^{\mathbb{C}}$, this isomorphism identifies the complex span $\left\langle\eta_{1}, \ldots, \eta_{k}\right\rangle^{\mathbb{C}}$ with the real span $\left\langle\operatorname{Re} \eta_{1}, \operatorname{Im} \eta_{1}, \ldots, \operatorname{Re} \eta_{k}, \operatorname{Im} \eta_{k}\right\rangle^{\mathbb{R}}$. When the structure is clear from the context, or is irrelevant, we shall continue to simply write $T^{*} \mathcal{U}$. We point out that the zero-section of $T^{*} \mathcal{U}$ is the conormal space to $\mathcal{U}$ in $\mathcal{U}$, i.e., $T_{\mathcal{U}}^{*} \mathcal{U}$.

We will projectivize the fibers of $\left(T^{*} \mathcal{U}\right)^{\mathbb{C}}$ (resp., $\left.\left(T^{*} \mathcal{U}\right)^{\mathbb{R}}\right)$, and denote this projectivization by $\mathbb{P}\left(\left(T^{*} \mathcal{U}\right)^{\mathbb{C}}\right)$ (resp., $\left.\mathbb{P}\left(\left(T^{*} \mathcal{U}\right)^{\mathbb{R}}\right)\right)$, which is isomorphic to $\mathcal{U} \times \mathbb{P}^{n}$ (resp., $\left.\mathcal{U} \times \mathbb{R}^{2 n+1}\right)$. In either the complex or real case, we let $\hat{\pi}$ denote the projection from the projectivization of $T^{*} \mathcal{U}$ to $\mathcal{U}$, and if $\eta$ is a non-zero element of the fiber $\left(T^{*} \mathcal{U}\right)_{p}$, we denote its projective class by $[\eta]$.

A subset $E \subseteq T^{*} \mathcal{U}$ is $\mathbb{C}$-conic (resp., $\mathbb{R}$-conic) if $(p, \eta) \in E$ implies that, for all $a \in \mathbb{C}$ (resp., $a \in$ $\mathbb{R}),(p, a \eta) \in E$. If $E$ is any subset of $T^{*} \mathcal{U}$, we let $\mathbb{P}(E)$ denote the (real or complex) projectivization $\left\{(p,[\eta]) \mid(p, \eta) \in E-T_{\mathcal{U}}^{*} \mathcal{U}\right\}$, and let $E_{p}:=\pi^{-1}(p) \cap E$.

We need the following easy lemmas.

Lemma 4.4. Suppose that $E \subseteq T^{*} \mathcal{U}$ is closed and $\mathbb{R}$-conic (resp., $\mathbb{C}$-conic). Then, $\mathbb{P}(E)$ is closed in $\mathbb{P}\left(\left(T^{*} \mathcal{U}\right)^{\mathbb{R}}\right)$ (resp., $\mathbb{P}\left(\left(T^{*} \mathcal{U}\right)^{\mathbb{C}}\right)$ ) and $\pi(E)$ is closed in $\mathcal{U}$.

Proof. We shall prove the real case. The proof over the complex numbers is the same. Throughout, we shall write simply $T^{*} \mathcal{U}$, in place of $\left(T^{*} \mathcal{U}\right)^{\mathbb{R}}$.

By definition of the quotient topology on $\mathbb{P}\left(T^{*} \mathcal{U}\right), \mathbb{P}(E)$ is closed if and only if

$$
E^{\prime}:=\left\{(p, \eta) \in T^{*} \mathcal{U}-T_{\mathcal{U}}^{*} \mathcal{U} \mid(p,[\eta]) \in \mathbb{P}(E)\right\}
$$

is closed in $T^{*} \mathcal{U}-T_{\mathcal{U}}^{*} \mathcal{U}$. As $E$ is conic, $E^{\prime}=E-T_{\mathcal{U}}^{*} \mathcal{U}$, which is closed in $T^{*} \mathcal{U}-T_{\mathcal{U}}^{*} \mathcal{U}$, since $E$ is closed in $T^{*} \mathcal{U}$. Thus, $\mathbb{P}(E)$ is closed.

Now, suppose that we have a sequence $p_{i} \in \pi(E)$ and $p_{i} \rightarrow p \in \mathcal{U}$. We need to show that $p \in \pi(E)$. Let $\eta_{i}$ be such that $\left(p_{i}, \eta_{i}\right) \in E$. Identify $T^{*} \mathcal{U}$ with $\mathcal{U} \times \mathbb{R}^{2 n+2}$.

If an infinite number of the $\eta_{i}$ are zero, then, by taking a subsequence (which we continue to write as $p_{i}$ ), we have an infinite sequence $\left(p_{i}, 0\right) \in E$. Then, $\left(p_{i}, 0\right) \rightarrow(p, 0) \in E$, as $E$ is closed. Thus, $p=\pi(p, 0) \in \pi(E)$.

If an infinite number of the $\eta_{i}$ are not zero, we can take a subsequence $\left(p_{i}, \eta_{i} /\left|\eta_{i}\right|\right)$, which is still in $E$, as $E$ is conic. Since the $\eta_{i} /\left|\eta_{i}\right|$ are contained in the unit sphere, by taking another subsequence, we may assume that $\eta_{i} /\left|\eta_{i}\right|$ converges to some $\eta$. Thus, $\left(p_{i}, \eta_{i} /\left|\eta_{i}\right|\right) \rightarrow(p, \eta)$, which is in $E$, since $E$ is closed, and so $p \in \pi(E)$.

Lemma 4.5. Suppose that $\tilde{h}_{1}, \ldots, \tilde{h}_{k}$ are real (resp., complex) analytic functions from $\mathcal{U}$ to $\mathbb{R}$ (resp., $\mathbb{C}$ ), and suppose that $E \subseteq T^{*} \mathcal{U}$ is closed and $\mathbb{R}$-conic (resp., $\mathbb{C}$-conic). Then, the set $\bar{\Sigma}_{E}\left(\tilde{h}_{1}, \ldots, \tilde{h}_{k}\right)$ of $p \in \mathcal{U}$ such that there exists non-zero $\left(a_{1}, \ldots, a_{k}\right) \in \mathbb{R}^{k}$ (resp., $\mathbb{C}^{k}$ ) such that

$$
a_{1} d_{p} \tilde{h}_{1}+\cdots+a_{k} d_{p} \tilde{h}_{k} \in E_{p}
$$

is closed in $\mathcal{U}$. 
Proof. We shall prove the real case. The proof over the complex numbers is the same. Let $N:=2 n+2$.

Let $K$ be the set of points $p \in \mathcal{U}$ such that $d_{p} \tilde{h}_{1}, \ldots, d_{p} \tilde{h}_{k}$ are linearly dependent, i.e., let $K$ be the critical locus of the map $\left(\tilde{h}_{1}, \ldots, \tilde{h}_{k}\right)$. Note that $K$ is closed.

Consider the continuous function $T:(\mathcal{U}-K) \times \mathbb{R} \mathbb{P}^{k-1} \rightarrow(\mathcal{U}-K) \times \mathbb{R} \mathbb{P}^{N-1}$ given by $T\left(p,\left[a_{1}, \ldots, a_{k}\right]\right)=$ $\left(p,\left[a_{1} d_{p} \tilde{h}_{1}+\cdots+a_{k} d_{p} \tilde{h}_{k}\right]\right)$. Let $\check{\pi}:(\mathcal{U}-K) \times \mathbb{R P P}^{k-1} \rightarrow \mathcal{U}-K$ denote the projection map.

By Lemma 4.4 $\mathbb{P}(E)$ is closed in $\mathcal{U} \times \mathbb{R} \mathbb{P}^{N-1}$. Therefore, $B:=T^{-1}\left(\left((\mathcal{U}-K) \times \mathbb{R} \mathbb{P}^{N-1}\right) \cap \mathbb{P}(E)\right)$ is closed in $(\mathcal{U}-K) \times \mathbb{R} \mathbb{P}^{k-1}$. As $\check{\pi}$ is proper, $\check{\pi}(B)$ is closed in $\mathcal{U}-K$.

Now, the set $\bar{\Sigma}_{E}\left(\tilde{h}_{1}, \ldots, \tilde{h}_{k}\right)$ is equal to $\check{\pi}(B) \cup K$, which is closed in $\mathcal{U}$.

Definition 4.6. Let $\widetilde{H}:=\left(\tilde{h}_{1}, \ldots, \tilde{h}_{k}\right)$ and $E$ be as in Lemma 4.5. Then, the set $\bar{\Sigma}_{E} \widetilde{H}$ from Lemma 4.5 is the closed E-critical locus of $\widetilde{H}$. We define the E-discriminant of $\widetilde{H}, \Delta_{E} \widetilde{H}$, to be $\widetilde{H}\left(\bar{\Sigma}_{E} \widetilde{H}\right)$.

If $\widetilde{H}$ is complex analytic, and $E=S S\left(\mathbf{F}^{\bullet}\right)$, then we let $\bar{\Sigma}_{\mathbf{F}} \cdot \widetilde{H}:=\bar{\Sigma}_{E} \widetilde{H}$ and $\Delta_{\mathbf{F}} \cdot \widetilde{H}:=\Delta_{E} \widetilde{H}$.

Remark 4.7. By Proposition 4.3 if $E:=S S\left(\mathbf{F}^{\bullet}\right)$, then $\overline{\Sigma_{\mathbf{F}} \bullet}=\bar{\Sigma}_{E} \tilde{f}$; this was our reason for adopting our notation for the closed $E$-critical locus.

While we shall not need it in this paper, it is possible to show that, in special cases, there is a reasonable notion of the (non-closed) critical locus $\Sigma_{E}\left(h_{1}, \ldots, h_{k}\right)$ which depends only on the restriction $\left(h_{1}, \ldots, h_{k}\right)$ of $\left(\tilde{h}_{1}, \ldots, \tilde{h}_{k}\right)$ to $\pi(E)$, and $\overline{\Sigma_{E}\left(h_{1}, \ldots, h_{k}\right)}=\bar{\Sigma}_{E}\left(\tilde{h}_{1}, \ldots, \tilde{h}_{k}\right)$. In particular, this is the case when $\widetilde{H}$ is complex analytic and $E=S S\left(\mathbf{F}^{\bullet}\right)$.

Lemma 4.8. Suppose that $f$ is not constant when restricted to $S \in \mathfrak{S}$. Then, for all $p \in \bar{S}$,

$$
\left(\overline{T_{S}^{*} \mathcal{U}}\right)_{p}+\left\langle d_{p} \tilde{f}\right\rangle:=\left\{\omega+a d_{p} \tilde{f} \mid a \in \mathbb{C}, \omega \in\left(\overline{T_{S}^{*} \mathcal{U}}\right)_{p}\right\} \subseteq\left(\overline{T_{f_{\mid S}^{*}}^{*} \mathcal{U}}\right)_{p}
$$

and, if $d_{p} \tilde{f} \notin\left(\overline{T_{S}^{*} \mathcal{U}}\right)_{p}$, then this containment is an equality.

Proof. Since $f$ is not constant on $S, S^{\prime}:=S-\Sigma\left(f_{\left.\right|_{S}}\right)$ is dense in $\bar{S}$. Thus, $\overline{T_{S}^{*} \mathcal{U}}=\overline{T_{S^{\prime}}^{*} \mathcal{U}}$, and $\overline{T_{f_{\mid S}^{*}}^{*} \mathcal{U}}=\overline{T_{f_{S^{\prime}}^{*}}^{*} \mathcal{U}}$.

Consider $\eta:=\omega+a d_{p} \tilde{f} \in\left(\overline{T_{S}^{*} \mathcal{U}}\right)_{p}+\left\langle d_{p} \tilde{f}\right\rangle$, where $\left(p_{i}, \omega_{i}\right) \in T_{S^{\prime}}^{*} \mathcal{U}$ and $\left(p_{i}, \omega_{i}\right) \rightarrow(p, \omega)$. Then, $\eta_{i}:=$ $\omega_{i}+a d_{p_{i}} \tilde{f} \in\left(T_{f_{\mid S}}^{*} \mathcal{U}\right)_{p_{i}}$, and $\eta_{i} \rightarrow \eta$. Therefore, the containment holds.

Suppose now that $d_{p} \tilde{f} \notin\left(\overline{T_{S}^{*} \mathcal{U}}\right)_{p}$, and that $\eta \in\left(\overline{T_{f_{\mid S}^{*}}^{*} \mathcal{U}}\right)_{p}=\left(\overline{T_{f_{S^{\prime}}}^{*} \mathcal{U}}\right)_{p}$. Then, there exists an analytic path $\left(p(t), \eta_{t}\right) \in \overline{T_{f_{S^{\prime}}}^{*} \mathcal{U}}$ such that $p=p(0), \eta=\eta_{0}$, and, for $t \neq 0,\left(p(0), \eta_{t}\right) \in T_{f_{S_{S^{\prime}}}}^{*} \mathcal{U}$. Hence, for $t \neq 0, \eta_{t}=\omega_{t}+\lambda(t) d_{p(t)} \tilde{f}$, where $\lambda(t) \in \mathbb{C}$ and $\omega_{t} \in\left(T_{S}^{*} \mathcal{U}\right)_{p(t)}$, and $\lambda(t)$ and $\omega_{t}$ are uniquely determined. Evaluating at $p^{\prime}(t)$, we find that, for $t \neq 0, \eta_{t}\left(p^{\prime}(t)\right)=\lambda(t) d_{p(t)} \tilde{f}\left(p^{\prime}(t)\right)$, and so $\lambda(t)$ is a quotient of two analytic functions. Therefore, there are two possibilities as $t \rightarrow 0$ : either $\lambda(t)$ approaches some $a \in \mathbb{C}$, or $|\lambda(t)| \rightarrow \infty$.

If $\lambda(t) \rightarrow a \in \mathbb{C}$, then $\omega_{t} \rightarrow \eta-a d_{p} \tilde{f}$, and $\eta=\left(\eta-a d_{p} \tilde{f}\right)+a d_{p} \tilde{f} \in\left(\overline{T_{S}^{*} \mathcal{U}}\right)_{p}+\left\langle d_{p} \tilde{f}\right\rangle$. We claim that $d_{p} \tilde{f} \notin\left(\overline{T_{S}^{*} \mathcal{U}}\right)_{p}$ implies that the case $|\lambda(t)| \rightarrow \infty$ cannot occur. Once we show this, the proof will be finished.

If $|\lambda(t)| \rightarrow \infty$, then, as $\eta_{t} \rightarrow \eta$

$$
\frac{\omega_{t}}{\lambda(t)}+d_{p(t)} \tilde{f}=\frac{\eta_{t}}{\lambda(t)} \rightarrow 0
$$


i.e.,

$$
d_{p} \tilde{f}=\lim _{t \rightarrow 0}\left(-\frac{\omega_{t}}{\lambda(t)}\right) \in\left(\overline{T_{S}^{*} \mathcal{U}}\right)_{p}
$$

\section{Lemma 4.9.}

1. $S S_{p}\left(\mathbf{F}^{\bullet}\right)+\left\langle d_{p} \tilde{f}\right\rangle \subseteq\left|\left(T_{f, \mathbf{F} \bullet}^{*} \mathcal{U}\right)^{\bullet}\right|_{p} \cup S S_{p}\left(\mathbf{F}^{\bullet}\right)$.

2. Suppose that $p \notin \overline{\Sigma_{\mathbf{F} \bullet f}}$. Then, $\left|\left(T_{f, \mathbf{F} \cdot}^{*} \mathcal{U}\right)^{\bullet}\right|_{p}=S S_{p}\left(\mathbf{F}^{\bullet}\right)+\left\langle d_{p} \tilde{f}\right\rangle$.

\section{Proof.}

Proof of Item 1:

Suppose that $\eta \in S S_{p}\left(\mathbf{F}^{\bullet}\right)+\left\langle d_{p} \tilde{f}\right\rangle$. Then, $\eta=\omega+a d_{p} \tilde{f}$, where $a \in \mathbb{C}$ and $\omega \in\left(\overline{T_{S}^{*} \mathcal{U}}\right)_{p}$ for some $S \in \mathfrak{S}\left(\mathbf{F}^{\bullet}\right)$. Then, there exist $\left(p_{i}, \omega_{i}\right) \in T_{S}^{*} \mathcal{U}$ such that $\left(p_{i}, \omega_{i}\right) \rightarrow(p, \omega)$. There are two cases.

If $f$ is constant on $S$, then $\left(p_{i}, \omega_{i}+a d_{p_{i}} \tilde{f}\right) \in T_{S}^{*} \mathcal{U}$. Hence, $\eta=\omega+a d_{p} \tilde{f} \in\left(\overline{T_{S}^{*} \mathcal{U}}\right)_{p} \subseteq S S_{p}\left(\mathbf{F}^{\bullet}\right)$. If $f$ is not constant on $S$, then Lemma 4.8 implies that $\eta \in\left|\left(T_{f, \mathbf{F} \cdot}^{*} \mathcal{U}\right)^{\bullet}\right|_{p}$.

Proof of Item 2:

Note that if $S \in \mathfrak{S}\left(\mathbf{F}^{\bullet}\right)$ and $f_{\left.\right|_{S}}$ is constant, then Proposition 4.3 implies that $S \subseteq \overline{\Sigma_{\mathbf{F}} \bullet}$; hence, by our hypothesis, $p \notin \bar{S}$. Therefore,

$$
S S_{p}\left(\mathbf{F}^{\bullet}\right)=\pi^{-1}(p) \cap \bigcup_{\substack{S \in \mathfrak{S}\left(\mathbf{F}^{\bullet}\right) \\ f_{\left.\right|_{S}} \neq \text { const. }}} \overline{T_{S}^{*} \mathcal{U}}
$$

Now the result follows immediately from Lemma 4.8 and the definition of $\left(T_{f, \mathbf{F}}^{*} \cdot \mathcal{U}\right)^{\bullet}$.

Proposition 4.10. There is an equality of sets

$$
\bar{\Sigma}_{\mathbf{F}} \cdot(\tilde{f}, \tilde{g})=\overline{\Sigma_{\mathbf{F}} \cdot f} \cup\left|\Gamma_{f, \tilde{g}}\left(\mathbf{F}^{\bullet}\right)\right| .
$$

Proof. Suppose that $p \notin \overline{\Sigma_{\mathbf{F} \bullet} f}$. Then, it follows immediately from Item 2 of Lemma 4.9 that $p \in \bar{\Sigma}_{\mathbf{F}} \bullet(\tilde{f}, \tilde{g})$ if and only if $p \in\left|\Gamma_{f, \tilde{g}}\left(\mathbf{F}^{\bullet}\right)\right|$. Therefore,

$$
\bar{\Sigma}_{\mathbf{F}}(\tilde{f}, \tilde{g})-\overline{\Sigma_{\mathbf{F}} \cdot f}=\left|\Gamma_{f, \tilde{g}}\left(\mathbf{F}^{\bullet}\right)\right|-\overline{\Sigma_{\mathbf{F}} \cdot f} .
$$

Now, take the union of both sides above with $\overline{\Sigma_{\mathbf{F}} \cdot f}$, and use that $\overline{\Sigma_{\mathbf{F}} \cdot f} \subseteq \bar{\Sigma}_{\mathbf{F}} \cdot(\tilde{f}, \tilde{g})$.

Remark 4.11. Note that is no claim in Proposition 4.10 about $\overline{\Sigma_{\mathbf{F}} \bullet f}$ and $\left|\Gamma_{f, \tilde{g}}\left(\mathbf{F}^{\bullet}\right)\right|$ intersecting in some nice way. In fact, in Remark 4.14 we give an example where these two sets are equal. However, if $\left|\Gamma_{f, \tilde{g}}\left(\mathbf{F}^{\bullet}\right)\right|$ is 1-dimensional and $f$ is not constant along any component of $\left|\Gamma_{f, \tilde{g}}\left(\mathbf{F}^{\bullet}\right)\right|$, then Proposition 4.3 implies that the intersection of $\overline{\Sigma_{\mathbf{F}} \cdot f}$ and $\left|\Gamma_{f, \tilde{g}}\left(\mathbf{F}^{\bullet}\right)\right|$ is either empty or consists of isolated points.

We also want to return to the topic of symmetry that we first discussed in Remark 3.5 By Proposition 4.10 and the symmetry of the definition of the closed critical locus, we have that

$$
\overline{\Sigma_{\mathbf{F}} \bullet f} \cup\left|\Gamma_{f, \tilde{g}}\left(\mathbf{F}^{\bullet}\right)\right|=\overline{\Sigma_{\mathbf{F}} g} \cup\left|\Gamma_{g, \tilde{f}}\left(\mathbf{F}^{\bullet}\right)\right| .
$$


By Proposition $4.3 f$ and $g$ are constant along the components of $\overline{\Sigma_{\mathbf{F}} \cdot f}$ and $\overline{\Sigma_{\mathbf{F}} \cdot g}$, respectively. It follows that the symmetric components of $\left|\Gamma_{f, \tilde{g}}\left(\mathbf{F}^{\bullet}\right)\right|$ and $\left|\Gamma_{g, \tilde{f}}\left(\mathbf{F}^{\bullet}\right)\right|$ are the same.

Note, however, that even in the classical case where we look at germs at the origin, $f$ is fixed, and $\tilde{g}$ is chosen to be a generic linear form, it is, in general, false that there is an equality of sets $\left|\Gamma_{f, \tilde{g}}\left(\mathbf{F}^{\bullet}\right)\right|=$ $\left|\Gamma_{g, \tilde{f}}\left(\mathbf{F}^{\bullet}\right)\right|$. Suppose, for instance, that $\operatorname{dim}_{\mathbf{0}} \overline{\Sigma_{\mathbf{F}} \cdot f} \geq 2$. For a generic linear form $\mathfrak{l}$, either $\overline{\Sigma_{\mathbf{F}} \bullet \mathfrak{l}}$ will be empty or the origin will be an isolated point in $\overline{\Sigma_{\mathbf{F}} \bullet}$; furthermore, Proposition 3.12 implies that $\left|\Gamma_{f, \tilde{g}}\left(\mathbf{F}^{\bullet}\right)\right|$ is purely 1-dimensional at the origin. However,

$$
\left|\Gamma_{g, \tilde{f}}\left(\mathbf{F}^{\bullet}\right)\right|=\overline{\Sigma_{\mathbf{F}} \cdot g} \cup\left|\Gamma_{g, \tilde{f}}\left(\mathbf{F}^{\bullet}\right)\right|=\overline{\Sigma_{\mathbf{F}} \bullet} \cup\left|\Gamma_{f, \tilde{g}}\left(\mathbf{F}^{\bullet}\right)\right|
$$

is, at least, 2-dimensional at the origin.

We now need to prove our main technical lemma.

Let $\stackrel{\circ}{\mathbb{D}}_{\delta}$ denote an open disk of radius $\delta$, centered at the origin, in $\mathbb{C}$. For positive $\rho, \delta, \epsilon \in \mathbb{R}$, let $N_{\delta, \rho}^{\epsilon}:=B_{\epsilon} \cap g^{-1}\left(\stackrel{\circ}{\mathbb{D}}_{\delta}\right) \cap f^{-1}\left(\stackrel{\circ}{\mathbb{D}}_{\rho}\right)$, let $P_{\delta, \rho}^{\epsilon}$ be the restriction of $(\tilde{g}, \tilde{f})$ to a map from $N_{\delta, \rho}^{\epsilon}$ to $\stackrel{\circ}{\mathbb{D}}_{\delta} \times \stackrel{\circ}{\mathbb{D}}_{\rho}$, and let $\left(\mathbf{F}^{\bullet}\right)_{\delta, \rho}^{\epsilon}$ denote the restriction of $\mathbf{F}^{\bullet}$ to $N_{\delta, \rho}^{\epsilon}$. Let $r: \mathcal{U} \rightarrow \mathbb{R}$ be the "squared distance from the origin" function $r=\left|z_{0}\right|^{2}+\cdots+\left|z_{n}\right|^{2}$.

Lemma 4.12. Suppose that $\operatorname{dim}_{\mathbf{0}} V(f) \cap\left|\Gamma_{f, \tilde{g}}\left(\mathbf{F}^{\bullet}\right)\right| \leq 0$. Then, there exists $\epsilon_{0}>0$ such that, for all $\epsilon_{1}$ and $\epsilon_{2}$ such that $0<\epsilon_{2}<\epsilon_{1} \leq \epsilon_{0}$, there exist $\delta, \rho>0$ such that

1. for all $p \in N_{\delta, \rho}^{\epsilon_{1}}-\stackrel{\circ}{B}_{\epsilon_{2}}$,

$$
d_{p} r \notin\left(S S_{p}\left(\mathbf{F}^{\bullet}\right)+<d_{p} \tilde{g}, d_{p} \tilde{f}>\right)^{\mathbb{R}}
$$

2. for all $p \in\left(N_{\delta, \rho}^{\epsilon_{1}}-\stackrel{\circ}{B}_{\epsilon_{2}}\right)-\overline{\Sigma_{\mathbf{F}} \cdot f}$, in particular, for all $p \in\left(N_{\delta, \rho}^{\epsilon_{1}}-\stackrel{\circ}{B}_{\epsilon_{2}}\right)-V(f)$, for all $(a, b) \in \mathbb{C}^{2}-\{\mathbf{0}\}$,

$$
\operatorname{Re}\left(a d_{p} \tilde{f}+b d_{p} \tilde{g}\right) \notin\left(S S_{p}\left(\mathbf{F}^{\bullet}\right)\right)^{\mathbb{R}}+\left\langle d_{p} r\right\rangle^{\mathbb{R}} .
$$

\section{Proof.}

Proof of Item 1:

Let $Y$ be the set of $p \in U$ such that there exists non-zero $(c, b) \in \mathbb{R} \times \mathbb{C}$ such that $c d_{p} r+\operatorname{Re}\left(b d_{p} \tilde{g}\right) \in$ $\left|\left(T_{f, \mathbf{F}}^{*} \mathcal{U}\right)^{\bullet}\right|_{p}^{\mathbb{R}}$. By Lemma 4.5 $Y$ is closed in $\mathcal{U}$. Let $Z$ be the set of $p \in U$ such that

$$
d_{p} r \in\left(S S_{p}\left(\mathbf{F}^{\bullet}\right)+<d_{p} \tilde{g}, d_{p} \tilde{f}>\right)^{\mathbb{R}} .
$$

Let $B_{\epsilon_{0}}^{*}:=B_{\epsilon_{0}}-\{\mathbf{0}\}$.

We shall prove Item 1 by proving that there exists $\epsilon_{0}>0$ such that

a. $B_{\epsilon_{0}}^{*} \cap Y \cap V(f, g)=\emptyset$;

b. $B_{\epsilon_{0}}^{*} \cap Z-V(f) \subseteq B_{\epsilon_{0}}^{*} \cap Y-V(f)$; and

c. for all $\epsilon_{1}, \epsilon_{2}$ such that $0<\epsilon_{2}<\epsilon_{1} \leq \epsilon_{0}$, there exists $\delta^{\prime}>0$ such that

$$
\left(B_{\epsilon_{1}}-\stackrel{\circ}{B}_{\epsilon_{2}}\right) \cap Z \cap g^{-1}\left(\stackrel{\circ}{\mathbb{D}}_{\delta^{\prime}}\right) \cap V(f)=\emptyset .
$$


We will first show how Items a, b, and c imply Item 1 . We will then show that Items a, b, and c hold.

Assume Items a, b, and c, and let $\epsilon_{1}, \epsilon_{2}$ be such that $0<\epsilon_{2}<\epsilon_{1} \leq \epsilon_{0}$. Let $\delta^{\prime}$ be as in Item c. As $Y$ is closed, $\left(B_{\epsilon_{1}}-\stackrel{\circ}{B}_{\epsilon_{2}}\right) \cap Y$ is compact. Thus, since $\left(B_{\epsilon_{1}}-\stackrel{\circ}{B}_{\epsilon_{2}}\right) \cap Y \cap V(f, g)=\emptyset$ by Item a, there exist $\rho, \delta^{\prime \prime}>0$ such that

$$
\left(B_{\epsilon_{1}}-\stackrel{\circ}{B}_{\epsilon_{2}}\right) \cap Y \cap g^{-1}\left(\stackrel{\circ}{\mathbb{D}}_{\delta^{\prime \prime}}\right) \cap f^{-1}\left(\stackrel{\circ}{\mathbb{D}}_{\rho}\right)=\emptyset .
$$

Fix such $\rho$ and $\delta^{\prime \prime}$. Fix $\delta$ such that $0<\delta \leq \min \left\{\delta^{\prime}, \delta^{\prime \prime}\right\}$. We wish to show that $\left(N_{\delta, \rho}^{\epsilon_{1}}-\stackrel{\circ}{B}_{\epsilon_{2}}\right) \cap Z=\emptyset$. Suppose that $p \in\left(N_{\delta, \rho}^{\epsilon_{1}}-\stackrel{\circ}{B}_{\epsilon_{2}}\right) \cap Z$. Then, $p \notin V(f)$ by Item c. However, then, Item b implies that $p \in Y$; a contradiction of $(\dagger)$.

Now we will show that we may pick $\epsilon_{0}>0$ so that Items $\mathrm{a}$, b, and $\mathrm{c}$ hold. Choose $\epsilon_{0}>0$ such that, for all $\epsilon$ such that $0<\epsilon \leq \epsilon_{0}, \partial B_{\epsilon}$ transversely intersects all of the strata of $\mathfrak{S}$. Then, for all $p \in B_{\epsilon_{0}}-\{\mathbf{0}\}$, $d_{p} r \notin\left(S S_{p}\left(\mathbf{F}^{\bullet}\right)\right)^{\mathbb{R}}$. Similarly, we may also choose $\epsilon_{0}>0$ so that, for all $\epsilon$ such that $0<\epsilon \leq \epsilon_{0}$, for all $p \in\left(B_{\epsilon_{0}}-\{\mathbf{0}\}\right)$,

$$
d_{p} r \notin\left(S S_{p}\left(\psi_{f}[-1] \mathbf{F}^{\bullet}\right)\right)^{\mathbb{R}} \cup\left(S S_{p}\left(\psi_{g}[-1] \mathbf{F}^{\bullet}\right)\right)^{\mathbb{R}} \cup\left(S S_{p}\left(\psi_{g}[-1] \psi_{f}[-1] \mathbf{F}^{\bullet}\right)\right)^{\mathbb{R}} .
$$

Combining the paragraph above with Proposition 4.3 and the equivalences at the beginning of Theorem 3.11 and using our hypothesis that $\operatorname{dim}_{\mathbf{0}} V(f) \cap\left|\Gamma_{f, \tilde{g}}\left(\mathbf{F}^{\bullet}\right)\right| \leq 0$, we may pick $\epsilon_{0}>0$ such that

i. $B_{\epsilon_{0}}^{*} \cap \bar{\Sigma}_{\mathbf{F}} \bullet f \subseteq V(f)$;

ii. $B_{\epsilon_{0}}^{*} \cap \bar{\Sigma}_{\psi_{f}[-1] \mathbf{F}} . g=\emptyset$;

iii. for all $p \in B_{\epsilon_{0}}^{*}$,

$$
d_{p} r \notin\left(S S_{p}\left(\mathbf{F}^{\bullet}\right)\right)^{\mathbb{R}} \cup\left(S S_{p}\left(\psi_{f}[-1] \mathbf{F}^{\bullet}\right)\right)^{\mathbb{R}} \cup\left(S S_{p}\left(\psi_{g}[-1] \mathbf{F}^{\bullet}\right)\right)^{\mathbb{R}} \cup\left(S S_{p}\left(\psi_{g}[-1] \psi_{f}[-1] \mathbf{F}^{\bullet}\right)\right)^{\mathbb{R}} .
$$

\section{Proof of Item a:}

Suppose that we have non-zero $(c, b) \in \mathbb{R} \times \mathbb{C}$ and $p \in B_{\epsilon_{0}}^{*} \cap V(f, g)$ such that $c d_{p} r+\operatorname{Re}\left(b d_{p} \tilde{g}\right) \in$ $\left|\left(T_{f, \mathbf{F}}^{*} \mathcal{U}\right)\right|_{p}^{\bullet}=\left(S S_{p}\left(\psi_{f}[-1] \mathbf{F}^{\bullet}\right)\right)^{\mathbb{R}}$, where the last equality follows from Theorem 3.7 If $c=0$, then $d_{p} \tilde{g} \in$ $S S_{p}\left(\psi_{f}[-1] \mathbf{F}^{\bullet}\right)$, i.e., $p \in \bar{\Sigma}_{\psi_{f}[-1] \mathbf{F}} \bullet$ $d_{p} r \in\left(S S_{p}\left(\psi_{f}[-1] \mathbf{F}^{\bullet}\right)+<d_{p} \tilde{g}>\right)^{\mathbb{R}}$. As $d_{p} \tilde{g} \notin S S_{p}\left(\psi_{f}[-1] \mathbf{F}^{\bullet}\right)$ and $p \in V(g)$, by Lemma 4.9 Item 2, $S S_{p}\left(\psi_{f}[-1] \mathbf{F}^{\bullet}\right)+<d_{p} \tilde{g}>=S S_{p}\left(\psi_{g}[-1] \psi_{f}[-1] \mathbf{F}^{\bullet}\right)$. However, $d_{p} r \in S S_{p}\left(\psi_{g}[-1] \psi_{f}[-1] \mathbf{F}^{\bullet}\right)$ contradicts Item iii. This proves Item a.

\section{Proof of Item b:}

Suppose that $p \in B_{\epsilon_{0}}^{*} \cap Z-V(f)$, i.e., $p \in B_{\epsilon_{0}}^{*}-V(f)$ and there exists $b \in \mathbb{C}$ such that $d_{p} r+\operatorname{Re}\left(b d_{p} \tilde{g}\right) \in$ $\left(S S_{p}\left(\mathbf{F}^{\bullet}\right)+<d_{p} \tilde{f}>\right)^{\mathbb{R}}$. By Item i and Lemma 4.9. Item $2, S S_{p}\left(\mathbf{F}^{\bullet}\right)+<d_{p} \tilde{f}>=\left|\left(T_{f, \mathbf{F}}^{*} \cdot \mathcal{U}\right)^{\bullet}\right|_{p}$, and so $d_{p} r+\operatorname{Re}\left(b d_{p} \tilde{g}\right) \in\left|\left(T_{f, \mathbf{F}}^{*} \cdot \mathcal{U}\right)\right|_{p}^{\mathbb{R}}$. Hence, $p \in Y$, and we have proved Item $\mathrm{b}$.

Proof of Item c: 
Let $W$ be the closed set

$$
W:=\left\{p \in \mathcal{U} \mid d_{p} r \in\left(\left|\left(T_{g, \psi_{f}[-1] \mathbf{F}^{\bullet} \mathbf{F}}^{*} \mathcal{U}\right)\right|_{p} \cup\left|\left(T_{g, \mathbf{F}}^{*} \mathcal{U}\right)^{\bullet}\right|_{p} \cup S S_{p}\left(\psi_{f}[-1] \mathbf{F}^{\bullet}\right) \cup S S_{p}\left(\mathbf{F}^{\bullet}\right)\right)^{\mathbb{R}}\right\} .
$$

By Item iii and Theorem 3.7 $B_{\epsilon_{0}}^{*} \cap W \cap V(g)=\emptyset$. We also claim that

$$
B_{\epsilon_{0}}^{*} \cap Z \cap V(f) \subseteq B_{\epsilon_{0}}^{*} \cap W \cap V(f) .
$$

For $p \in Z$ if and only if there exists a non-zero $b \in \mathbb{C}$ such that $d_{p} r+\operatorname{Re}\left(b d_{p} \tilde{g}\right) \in\left(S S_{p}\left(\mathbf{F}^{\bullet}\right)+<d_{p} \tilde{f}>\right)^{\mathbb{R}}$, and, by Item 1 of Lemma 4.9

$$
S S_{p}\left(\mathbf{F}^{\bullet}\right)+<d_{p} \tilde{f}>\subseteq\left|\left(T_{f, \mathbf{F}}^{*} \mathcal{U}\right)^{\bullet}\right|_{p} \cup S S_{p}\left(\mathbf{F}^{\bullet}\right) .
$$

If $p \in V(f)$, then Theorem 3.7 implies that $\left|\left(T_{f, \mathbf{F}}^{*} \mathcal{U}\right)^{\bullet}\right|_{p}=S S_{p}\left(\psi_{f}[-1] \mathbf{F}^{\bullet}\right)$. Now, (†) follows at once.

Let $\epsilon_{1}, \epsilon_{2}$ be such that $0<\epsilon_{2}<\epsilon_{1} \leq \epsilon_{0}$. Then, since $B_{\epsilon_{0}}^{*} \cap W \cap V(g)=\emptyset$ and $W$ is closed, $\left(B_{\epsilon_{1}}-\stackrel{\circ}{B_{\epsilon_{2}}}\right) \cap W$ is compact and $\left(B_{\epsilon_{1}}-\stackrel{\circ}{B}_{\epsilon_{2}}\right) \cap W \cap V(g)=\emptyset$. Thus, there exists $\delta^{\prime}>0$ such that $\left(B_{\epsilon_{1}}-\stackrel{\circ}{B}_{\epsilon_{2}}\right) \cap W \cap g^{-1}\left(\stackrel{\circ}{\mathbb{D}}_{\delta^{\prime}}\right)=\emptyset$. Finally, we conclude that

$$
\left(B_{\epsilon_{1}}-\stackrel{\circ}{B}_{\epsilon_{2}}\right) \cap Z \cap g^{-1}\left(\stackrel{\circ}{\mathbb{D}}_{\delta^{\prime}}\right) \cap V(f) \subseteq\left(B_{\epsilon_{1}}-\stackrel{\circ}{B}_{\epsilon_{2}}\right) \cap W \cap g^{-1}\left(\stackrel{\circ}{\mathbb{D}}_{\delta^{\prime}}\right) \cap V(f)=\emptyset,
$$

which proves Item c, and concludes the proof of Item 1 from the statement of the lemma.

Proof of Item 2:

Assume that $\operatorname{dim}_{\mathbf{0}} V(f) \cap\left|\Gamma_{f, \tilde{g}}\left(\mathbf{F}^{\bullet}\right)\right| \leq 0$. Pick $\epsilon_{0}>0$ so that $B_{\epsilon_{0}} \cap V(f) \cap\left|\Gamma_{f, \tilde{g}}\left(\mathbf{F}^{\bullet}\right)\right| \subseteq\{\mathbf{0}\}$. This means precisely that

$$
\left(B_{\epsilon_{0}}-\{\mathbf{0}\}\right) \cap\left\{\left.p \in \mathcal{U}\left|d_{p} \tilde{g} \in\right|\left(T_{f, \mathbf{F} \cdot}^{*} \mathcal{U}\right)\right|_{p}\right\} \cap V(f)=\emptyset .
$$

Let $\epsilon_{1}$ and $\epsilon_{2}$ be such that $0<\epsilon_{2}<\epsilon_{1} \leq \epsilon_{0}$, and assume that $\rho$ and $\delta$ are such that Item 1 holds. Then,

$$
\left(B_{\epsilon_{1}}-\stackrel{\circ}{B}_{\epsilon_{2}}\right) \cap V(f) \cap\left|\Gamma_{f, \tilde{g}}\left(\mathbf{F}^{\bullet}\right)\right|=\emptyset .
$$

As $\left(B_{\epsilon_{1}}-\stackrel{\circ}{B}_{\epsilon_{2}}\right) \cap\left|\Gamma_{f, \tilde{g}}\left(\mathbf{F}^{\bullet}\right)\right|$ is compact, it follows that we may re-choose $\rho$, smaller if needed, so that

$$
\left(B_{\epsilon_{1}}-\stackrel{\circ}{B}_{\epsilon_{2}}\right) \cap f^{-1}\left(\stackrel{\circ}{\mathbb{D}}_{\rho}\right) \cap\left|\Gamma_{f, \tilde{g}}\left(\mathbf{F}^{\bullet}\right)\right|=\emptyset .
$$

We claim that Item 2 holds.

To see this, let $p \in\left(N_{\delta, \rho}^{\epsilon_{1}}-\stackrel{\circ}{B}_{\epsilon_{2}}\right)-\overline{\Sigma_{\mathbf{F} \bullet} f}$, and assume that we have $(a, b) \in \mathbb{C}^{2}-\{\mathbf{0}\}$ such that

$$
\operatorname{Re}\left(a d_{p} \tilde{f}+b d_{p} \tilde{g}\right) \in\left(S S_{p}\left(\mathbf{F}^{\bullet}\right)\right)^{\mathbb{R}}+\left\langle d_{p} r\right\rangle^{\mathbb{R}} .
$$

Then, there exists a real number $c$ such that

$$
c d_{p} r+\operatorname{Re}\left(a d_{p} \tilde{f}+b d_{p} \tilde{g}\right) \in\left(S S_{p}\left(\mathbf{F}^{\bullet}\right)\right)^{\mathbb{R}} .
$$

If $c \neq 0$, we may divide by $c$ and obtain a contradiction to Item 1 . Thus, $c$ must equal 0 , and so

$$
a d_{p} \tilde{f}+b d_{p} \tilde{g} \in S S_{p}\left(\mathbf{F}^{\bullet}\right)
$$


i.e., $p \in \bar{\Sigma}_{\mathbf{F}} \bullet(\tilde{f}, \tilde{g})$, which by Proposition 4.10 is equal to $\overline{\Sigma_{\mathbf{F}} \bullet f} \cup\left|\Gamma_{f, \tilde{g}}\left(\mathbf{F}^{\bullet}\right)\right|$. This is a contradiction of (†) and the fact that $p \notin \overline{\Sigma_{\mathbf{F} \bullet} f}$.

As before, for $\epsilon, \delta, \rho>0$, let $N_{\delta, \rho}^{\epsilon}:=B_{\epsilon} \cap g^{-1}\left(\stackrel{\circ}{\mathbb{D}}_{\delta}\right) \cap f^{-1}\left(\stackrel{\circ}{\mathbb{D}}_{\rho}\right)$. Let $\left(\mathbf{F}^{\bullet}\right)_{\delta, \rho}^{\epsilon}$ be the restriction of $\mathbf{F}^{\bullet}$ to $N_{\delta, \rho}^{\epsilon}$, and let $T_{\delta, \rho}^{\epsilon}$ be the restriction of the map $(g, f)$ to a map from $N_{\delta, \rho}^{\epsilon}$ to $\stackrel{\circ}{\mathbb{D}}_{\delta} \times \stackrel{\circ}{\mathbb{D}}_{\rho}$.

Theorem 4.13. (The Derived Category Discriminant Theorem) Suppose that $\operatorname{dim}_{\mathbf{0}} V(f) \cap\left|\Gamma_{f, \tilde{g}}\left(\mathbf{F}^{\bullet}\right)\right| \leq 0$.

Then, for all sufficiently small $\epsilon>0$, there exist $\delta, \rho>0$ such that the derived push-forward $\mathbf{A}^{\bullet}:=$ $R\left(T_{\delta, \rho}^{\epsilon}\right)_{*}\left(\mathbf{F}^{\bullet}\right)_{\delta, \rho}^{\epsilon}$ is complex analytically constructible with respect to the stratification given by

$$
\left\{\stackrel{\circ}{\mathbb{D}}_{\delta} \times \stackrel{\circ}{\mathbb{D}}_{\rho}-\Delta_{\mathbf{F}} \cdot(\tilde{g}, \tilde{f}),\left(\stackrel{\circ}{\mathbb{D}}_{\delta} \times \stackrel{\circ}{\mathbb{D}}_{\rho}\right) \cap \Delta_{\mathbf{F}} \cdot(\tilde{g}, \tilde{f})-\{\mathbf{0}\},\{\mathbf{0}\}\right\}
$$

Proof. Fix choices of $\epsilon_{0}, \epsilon_{1}, \epsilon_{2}, \delta$, and $\rho$ as in Lemma 4.12 Pick $\epsilon$ so that $\epsilon_{2}<\epsilon<\epsilon_{1}$. Let $\mathbf{G}^{\bullet}$ be the restriction of $\mathbf{F}^{\bullet}$ to $Y:=\stackrel{\circ}{B}_{\epsilon_{1}} \cap g^{-1}\left(\stackrel{\circ}{\mathbb{D}}_{\delta}\right) \cap f^{-1}\left(\stackrel{\circ}{\mathbb{D}}_{\rho}\right)$. Let $\tilde{h}$ be the restriction of $(g, f)$ to a map from $Y$ to $\stackrel{\circ}{\mathbb{D}}_{\delta} \times \stackrel{\circ}{\mathbb{D}}_{\rho}$. Then, by applying Item 1 of Lemma 4.12 and Proposition 8.5.8 of $[6]$ to $\mathbf{G}^{\bullet}$ (where the $\phi$ and $f$ of [6] are our $r$ and $(g, f)$, respectively), we immediately conclude that $\mathbf{A}^{\bullet}$ is complex analytically constructible.

As $\Delta_{\mathbf{F}} \cdot(\tilde{g}, \tilde{f})$ is either empty or a curve, to show that $\mathbf{A}^{\bullet}$ is constructible with respect to the given stratification, one has only to show that the cohomology of $\mathbf{A}^{\bullet}$ is locally constant at points in $\stackrel{\circ}{\mathbb{D}}_{\delta} \times \stackrel{\circ}{\mathbb{D}}_{\rho}-$ $\Delta_{\mathbf{F}} \bullet(\tilde{g}, \tilde{f})$. Let $q:=\left(u_{0}, v_{0}\right) \in \stackrel{\circ}{\mathbb{D}}_{\delta} \times \stackrel{\circ}{\mathbb{D}}_{\rho} ;$ it suffices to show that $S S_{q}\left(\mathbf{A}^{\bullet}\right)=\{0\}$.

In the following, we use the real structure in each of the statements. Proposition 8.5.8 of [6] implies Proposition 5.4 .17 of [6]. Item ii, part d, of this latter proposition tells us that

$$
S S_{q}\left(\mathbf{A}^{\bullet}\right) \subseteq \bigcup_{p \in B_{\epsilon} \cap g^{-1}\left(u_{0}\right) \cap f^{-1}\left(v_{0}\right)}\left\{a d_{p} \tilde{g}+b d_{p} \tilde{f} \in S S_{p}\left(\left(\mathbf{F}^{\bullet}\right)_{\delta, \rho}^{\epsilon}\right) \mid(a, b) \in \mathbb{C}^{2}\right\} .
$$

If $p \in \stackrel{\circ}{B}_{\epsilon}$, then $S S_{p}\left(\left(\mathbf{F}^{\bullet}\right)_{\delta, \rho}^{\epsilon}\right)=S S_{p}\left(\mathbf{F}^{\bullet}\right)$, and so $\left\{a d_{p} \tilde{g}+b d_{p} \tilde{f} \in S S_{p}\left(\left(\mathbf{F}^{\bullet}\right)_{\delta, \rho}^{\epsilon}\right) \mid(a, b) \in \mathbb{C}^{2}\right\}=\{0\}$ as $p \notin \bar{\Sigma}_{\mathbf{F}} \bullet(\tilde{f}, \tilde{g})$. If $p \in \partial B_{\epsilon}$, then, by Proposition 5.4 .8 of [6], $S S_{p}\left(\left(\mathbf{F}^{\bullet}\right)_{\delta, \rho}^{\epsilon}\right) \subseteq S S_{p}\left(\mathbf{F}^{\bullet}\right)+<d_{p} r>$, and so $\left\{a d_{p} \tilde{g}+b d_{p} \tilde{f} \in S S_{p}\left(\left(\mathbf{F}^{\bullet}\right)_{\delta, \rho}^{\epsilon}\right) \mid(a, b) \in \mathbb{C}^{2}\right\}=\{0\}$ by Item 2 of Lemma 4.12

Remark 4.14. The assumption that $\operatorname{dim}_{0} V(f) \cap\left|\Gamma_{f, \tilde{g}}\left(\mathbf{F}^{\bullet}\right)\right| \leq 0$ is crucial in Lemma 4.12 and Theorem4.13 Consider the classic example of the map $H:=(\tilde{g}, \tilde{f})=(g, f): \mathbb{C}^{3} \rightarrow \mathbb{C}^{2}$ given by $g(x, y, t)=x$ and $f(x, y, t)=y^{2}-t x^{2}$, where it is not possible to stratify the domain and codomain in order to obtain a Thom map. The (ordinary) discriminant of $H$ is simply the origin and, yet, for $0<\delta, \rho \ll \epsilon \ll 1$, the isomorphismtype of the cohomology of the fibers $B_{\epsilon} \cap H^{-1}(a, b)$ is not independent of the choice of $(a, b) \in \stackrel{\circ}{\mathbb{D}}_{\delta} \times \stackrel{\circ}{\mathbb{D}}_{\rho}-\{\mathbf{0}\}$.

The reader should verify that, in this example, $\left|\Gamma_{f, \tilde{g}}\left(\mathbf{F}^{\bullet}\right)\right|=V(x, y)$ and so the condition that $\operatorname{dim}_{\mathbf{0}} V(f) \cap$ $\left|\Gamma_{f, \tilde{g}}\left(\mathbf{F}^{\bullet}\right)\right| \leq 0$ does not hold.

Corollary 4.15. Suppose that $\operatorname{dim}_{\mathbf{0}} V(f) \cap\left|\Gamma_{f, \tilde{g}}\left(\mathbf{F}^{\bullet}\right)\right| \leq 0$. Let $\left(\widehat{\Gamma}_{f, \tilde{g}}\left(\mathbf{F}^{\bullet}\right)\right)^{\bullet}$ denote the components of $\left(\Gamma_{f, \tilde{g}}\left(\mathbf{F}^{\bullet}\right)\right)^{\bullet}$ which are not contained in $V(g)$. Then,

1.

$$
\mathbb{H}^{k-1}\left(F_{f, \mathbf{0}}, F_{f_{\left.\right|_{V}(g)}, \mathbf{0}} ; \mathbf{F}^{\bullet}\right) \cong\left(\left(\widehat{\Gamma}_{f, \tilde{g}}\left(\mathbf{F}^{\bullet}\right)\right)^{k} \odot V(f)\right)_{\mathbf{0}}
$$


2.

$$
\mathbb{H}^{k-1}\left(F_{g, \mathbf{0}}, F_{g_{\left.\right|_{V}(f)}, \mathbf{0}} ; \mathbf{F}^{\bullet}\right) \cong\left(\left(\widehat{\Gamma}_{f, \tilde{g}}\left(\mathbf{F}^{\bullet}\right)\right)^{k} \odot V(g)\right)_{\mathbf{0}} ; \text { and }
$$

3.

$$
H^{k}\left(\phi_{f}[-1] \psi_{g}[-1] \mathbf{F}^{\bullet}\right)_{\mathbf{0}} \cong\left(\left(\widehat{\Gamma}_{f, \tilde{g}}\left(\mathbf{F}^{\bullet}\right)\right)^{k} \odot V(g)\right)_{\mathbf{0}} \oplus H^{k}\left(\psi_{g}[-1] \phi_{f}[-1] \mathbf{F}^{\bullet}\right)_{\mathbf{0}}
$$

Proof. Now that we have Theorem 4.13 the proof of each item is obtained by looking at the relative hypercohomology of a complex disk modulo a point, and using that this relative hypercohomology splits as a direct sum. One "sees" the results by looking at "pictures" in $\stackrel{\circ}{\mathbb{D}}_{\delta} \times \stackrel{\circ}{\mathbb{D}}_{\rho}$; exactly as in the case where $\mathbf{F}^{\bullet}$ is the constant sheaf on affine space and $g$ is a generic linear form. The discriminant/Cerf diagram arguments remain the same, except that it is no longer true that the components of the Cerf diagram are tangent to the horizontal axis at the origin, i.e., it is not necessarily true for each component $C$ of $\left|\left(\widehat{\Gamma}_{f, \tilde{g}}\left(\mathbf{F}^{\bullet}\right)\right)^{\bullet}\right|$ that $(C \cdot V(f))_{\mathbf{0}}>(C \cdot V(g))_{\mathbf{0}}$.

Of course, the pictures are actually drawn in $\mathbb{R}^{2}$, and so a line segment represents a complex disk (but a point still represents a point). The three relevant pictures, in order, are:
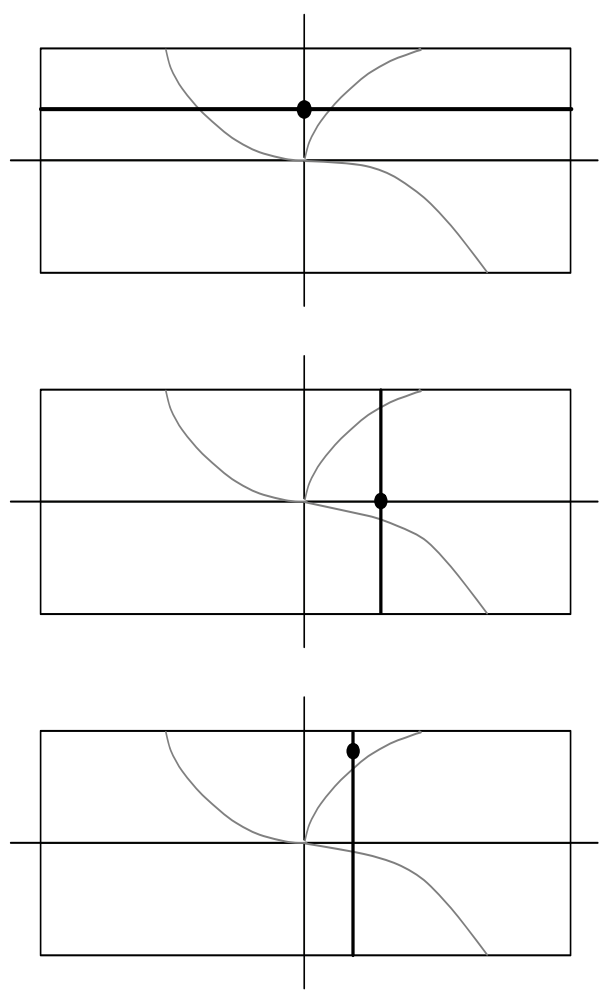

Remark 4.16. If $\operatorname{dim}_{\mathbf{0}} V(g) \cap\left|\Gamma_{f, \tilde{g}}\left(\mathbf{F}^{\bullet}\right)\right| \leq 0$, then $\left(\widehat{\Gamma}_{f, \tilde{g}}\left(\mathbf{F}^{\bullet}\right)\right)^{\bullet}=\left(\Gamma_{f, \tilde{g}}\left(\mathbf{F}^{\bullet}\right)\right)^{\bullet}$, and Item 1 of Corollary 4.15] combined with Theorem 3.11] yields an isomorphism between the cohomology $\mathbb{H}^{*}\left(F_{f, \mathbf{0}}, F_{f_{\left.\right|_{V(g)}}, \mathbf{0}} ; \mathbf{F}^{\bullet}[-1]\right)$ and $H^{*}\left(\phi_{g}[-1] \psi_{f}[-1] \mathbf{F}^{\bullet}\right)_{\mathbf{0}}$; this isomorphism is not natural. In particular, the Milnor monodromy of $f$ typically induces completely different automorphisms on these two cohomologies. 
Corollary 4.17. The following are equivalent:

1. $\mathbf{0} \notin\left|\Gamma_{f, \tilde{g}}\left(\mathbf{F}^{\bullet}\right)\right|$;

2. for all $p \in V(f, g)$ near the origin, $H^{*}\left(\phi_{g}[-1] \psi_{f}[-1] \mathbf{F}^{\bullet}\right)_{p}=0$;

3. $\operatorname{dim}_{\mathbf{0}} V(f) \cap\left|\Gamma_{f, \tilde{g}}\left(\mathbf{F}^{\bullet}\right)\right| \leq 0$, and $H^{*}\left(\phi_{g}[-1] \psi_{f}[-1] \mathbf{F}^{\bullet}\right)_{\mathbf{0}}=0$;

4. $\operatorname{dim}_{\mathbf{0}} V(g) \cap\left|\Gamma_{f, \tilde{g}}\left(\mathbf{F}^{\bullet}\right)\right| \leq 0$, and inclusion induces an isomorphism between $\mathbb{H}^{*}\left(F_{f, \mathbf{0}} ; \mathbf{F}^{\bullet}\right)$ and $\mathbb{H}^{*}\left(F_{f_{\left.\right|_{V(g)}}}, \mathbf{0} ; \mathbf{F}^{\bullet}\right)$;

5. $\operatorname{dim}_{\mathbf{0}} V(g) \cap\left|\Gamma_{f, \tilde{g}}\left(\mathbf{F}^{\bullet}\right)\right| \leq 0$, and inclusion induces an isomorphism between $\mathbb{H}^{*}\left(F_{g, \mathbf{0}} ; \mathbf{F}^{\bullet}\right)$ and $\mathbb{H}^{*}\left(F_{g_{\mid}(f)}, \mathbf{0} ; \mathbf{F}^{\bullet}\right)$;

6. $\operatorname{dim}_{\mathbf{0}} V(g) \cap\left|\Gamma_{f, \tilde{g}}\left(\mathbf{F}^{\bullet}\right)\right| \leq 0$, and $H^{*}\left(\phi_{f}[-1] \psi_{g}[-1] \mathbf{F}^{\bullet}\right)_{\mathbf{0}}$ is isomorphic to $H^{*}\left(\psi_{g}[-1] \phi_{f}[-1] \mathbf{F}^{\bullet}\right)_{\mathbf{0}}$.

Proof. This is immediate from Lemma 3.10] Theorem 3.11 and Corollary 4.15]

The above corollary has a more familiar feel in the classical case, where $\tilde{g}$ is a generic linear form. Recall that, in Proposition 3.12 we showed that, if $\mathfrak{l}$ is a generic linear form on $\mathcal{U}$, then, $\operatorname{dim}_{\mathbf{0}} V(f) \cap\left|\Gamma_{f, \mathfrak{l}}\left(\mathbf{F}^{\bullet}\right)\right| \leq 0$ and $\operatorname{dim}_{\mathbf{0}} V(\mathfrak{l}) \cap\left|\Gamma_{f, \mathfrak{l}}\left(\mathbf{F}^{\bullet}\right)\right| \leq 0$. Thus, Corollary 4.17 immediately yields:

Corollary 4.18. The following are equivalent:

1. there exists a non-zero linear form $\mathfrak{l}$ such that $\mathbf{0} \notin\left|\Gamma_{f, \mathfrak{l}}\left(\mathbf{F}^{\bullet}\right)\right|$;

2. for generic linear $\mathfrak{l}, \mathbf{0} \notin\left|\Gamma_{f, \mathfrak{l}}\left(\mathbf{F}^{\bullet}\right)\right|$;

3. for generic linear $\mathfrak{l}, H^{*}\left(\phi_{\mathrm{r}}[-1] \psi_{f}[-1] \mathbf{F}^{\bullet}\right)_{\mathbf{0}}=0$;

4. for generic linear $\mathfrak{l}$, inclusion induces an isomorphism between $\mathbb{H}^{*}\left(F_{f, \mathbf{0}} ; \mathbf{F}^{\bullet}\right)$ and $\mathbb{H}^{*}\left(F_{f_{\left.\right|_{V(l)}}, \mathbf{0}} ; \mathbf{F}^{\bullet}\right)$;

5. for generic linear $\mathfrak{l}$, inclusion induces an isomorphism between $\mathbb{H}^{*}\left(F_{\mathfrak{l}, \mathbf{0}} ; \mathbf{F}^{\bullet}\right)$ and $\mathbb{H}^{*}\left(F_{\mathfrak{l}_{\left.\right|_{V}(f)}}, \mathbf{0} ; \mathbf{F}^{\bullet}\right)$;

6. for generic linear $\mathfrak{l}, H^{*}\left(\phi_{f}[-1] \psi_{\mathfrak{l}}[-1] \mathbf{F}^{\bullet}\right)_{\mathbf{0}}$ is isomorphic to $H^{*}\left(\psi_{\mathfrak{l}}[-1] \phi_{f}[-1] \mathbf{F}^{\bullet}\right)_{\mathbf{0}}$.

Remark 4.19. Suppose that $X=\mathcal{U}$ and $\mathbf{F}^{\bullet}=\mathbb{Z}_{\mathcal{U}}$ throughout this remark.

Then, $\mathbb{H}^{*}\left(F_{\mathfrak{l}, \mathbf{0}} ; \mathbf{F}^{\bullet}\right)$ has the cohomology of a point and $\mathbb{H}^{*}\left(F_{\mathfrak{l}_{\mid V(f)}}, \mathbf{0} ; \mathbf{F}^{\bullet}\right)$ is the cohomology of the complex link of $V(f)$ at $\mathbf{0}$. Hence, the equivalence of Item 2 (or Item 1) and Item 5 of Corollary 4.18 is a generalization of the well-known result that the cohomology of the complex link of $V(f)$ at $\mathbf{0}$ is isomorphic to that of a point if and only if the relative polar curve is empty (in a neighborhood of $\mathbf{0}$ ).

Suppose now that we also know have that $\operatorname{dim}_{0} \Sigma f=1$. Then, for generic $\mathfrak{l}, f_{\left.\right|_{V(\mathrm{I})}}$ has an isolated critical point at the origin, and $H^{*}\left(\phi_{f}[-1] \psi_{r}[-1] \mathbf{F}^{\bullet}\right)_{\mathbf{0}}$ is isomorphic to the (shifted) reduced integral cohomology of the Milnor fiber at the origin of $f_{\left.\right|_{V(r)}}$. On the other hand, $H^{*}\left(\psi_{\mathfrak{r}}[-1] \phi_{f}[-1] \mathbf{F}^{\bullet}\right)_{\mathbf{0}}$ is isomorphic to the direct sum of the reduced integral cohomologies of the Milnor fibers of $f_{\left.\right|_{V(\mathbb{I}-t)}}$, where the sum is over all points $p \in \stackrel{\circ}{B}_{\epsilon} \cap \Sigma f \cap V(\mathfrak{l}-t)$ for $0<|t| \ll \epsilon \ll 1$. Therefore, the equivalence of Item 2 (or Item 1) and Item 6 of Corollary 4.18 is a generalization of the well-known result that the Milnor number of a generic hyperplane slice equals the sum of the Milnor numbers in a nearby hyperplane slice if and only if the relative polar curve is empty (in a neighborhood of $\mathbf{0}$ ). 


\section{An Application to Thom's $a_{f}$ Condition}

By combining our results from [18] with Theorem 3.11 we can relate the polar curve to Thom's $a_{f}$ condition. Essentially what we prove below, in Theorem [5.6] is that, if a stratification satisfies the $a_{f}$ condition, except perhaps at a point $p$ on a 1-dimensional stratum, then the stratification satisfies the $a_{f}$ condition at $p$ if and only if, for some affine linear form $\mathfrak{l}$, the polar curve of $(f, \mathfrak{l})$ at $p$ is empty.

However, we do not actually need to start with a stratification, for we do not need the condition of the frontier. Also, of course, we want such a result with respect to a complex of sheaves. So, we need to make a number of preliminary definitions before we can state and prove our precise result.

Suppose that $M$ and $N$ are complex submanifolds of $\mathcal{U}$.

Definition 5.1. The pair $(M, N)$ satisfies Thom's $a_{\tilde{f}}$ condition at a point $x \in N$ if and only if there is an inclusion, of fibers over $x,\left(\overline{T_{\tilde{f}_{\mid M}}^{*} \mathcal{U}}\right)_{x} \subseteq\left(T_{\tilde{f}_{\mid}}^{*} \mathcal{U}\right)_{x}$.

The pair $(M, N)$ satisfies Thom's $a_{\tilde{f}}$ condition if and only if it satisfies the $a_{\tilde{f}}$ condition at each point $x \in N$.

Remark 5.2. Note that if $\tilde{f}$ is a locally constant function, then the $a_{\tilde{f}}$ condition reduces to condition (a) of Whitney.

The $a_{\tilde{f}}$ is condition is important for several reasons. First, it is an hypothesis of Thom's second isotopy lemma; see [19]. Second, the $a_{\tilde{f}}$ condition, and the existence of stratifications in which all pairs of strata satisfy the $a_{\tilde{f}}$ condition, is essential in arguments such as that used by Lê in $\underline{9}$ to prove that Milnor fibrations exist even when the domain is an arbitrarily singular space. Third, the $a_{\tilde{f}}$ condition is closely related to constancy of the Milnor number in families of isolated hypersurface singularities; see [11].

There are at least two important general results about the $a_{\tilde{f}}$ condition: the above-mentioned existence of $a_{\tilde{f}}$ stratifications, proved first by Hironaka in [5] and then in a different manner by Hamm and Lê, following an argument of F. Pham, in Theorem 1.2.1 of [4], and the fact that Whitney stratifications in which $V(\tilde{f}):=\tilde{f}^{-1}(0)$ is a union of strata are $a_{\tilde{f}}$ stratifications, proved independently by Parusiński in [20], and Briançon, P. Maisonobe, and M. Merle in [1].

Definition 5.3. A collection $\mathcal{W}$ of subsets of $X$ is a (complex analytic) partition of $X$ if and only if $\mathcal{W}$ is a locally finite disjoint collection of analytic submanifolds of $\mathcal{U}$, which we call strata, whose union is all of $X$, and such that, for each stratum $W \in \mathcal{W}, \bar{W}$ and $\bar{W}-W$ are closed complex analytic subsets of $X$.

In this paper, we assume that all of the strata of a partition are connected.

A partition $\mathcal{W}$ is a stratification if and only if it satisfies the condition of the frontier, i.e., for all $W \in \mathcal{W}, \bar{W}$ is a union of elements of $\mathcal{W}$.

Below, we extend our earlier definition of $\mathbf{F}^{\bullet}$-visible strata to the case of a partition which may not satisfy Whitney conditions.

Definition 5.4. A partition $\mathcal{W}$ of $X$ is an $\mathbf{F}^{\bullet}$-partition provided that

$$
S S\left(\mathbf{F}^{\bullet}\right) \subseteq \bigcup_{W \in \mathcal{W}} \overline{T_{W}^{*} \mathcal{U}}
$$


If $\mathcal{W}$ is an $\mathbf{F}^{\bullet}$-partition, then a stratum $W \in \mathcal{W}$ is $\mathbf{F}^{\bullet}$-visible if and only if $\overline{T_{W}^{*} \mathcal{U}} \subseteq S S\left(\mathbf{F}^{\bullet}\right)$. We let $\mathcal{W}\left(\mathbf{F}^{\bullet}\right):=\left\{W \in \mathcal{W} \mid W\right.$ is $\mathbf{F}^{\bullet}$-visible $\}$.

Remark 5.5. The reader should understand that the point of an $\mathbf{F}^{\bullet}$-partition $\mathcal{W}$ is that, for each $\mathbf{F}^{\bullet}$-visible stratum $S$ in $\mathfrak{S}$, there exists a unique $W \in \mathcal{W}$ such that $\bar{S}=\bar{W}$ and, hence, $\overline{T_{S}^{*} \mathcal{U}}=\overline{T_{W}^{*} \mathcal{U}}$. It follows at once from this, and the definition of $\mathbf{F}^{\bullet}$-visible strata of $\mathcal{W}$, that, if $\mathcal{W}$ is an $\mathbf{F}^{\bullet}$-partition, then

$$
S S\left(\mathbf{F}^{\bullet}\right)=\bigcup_{W \in \mathcal{W}\left(\mathbf{F}^{\bullet}\right)} \overline{T_{W}^{*} \mathcal{U}}
$$

We can now give our result on Thom's $a_{f}$ condition and the relative polar curve.

Theorem 5.6. Suppose that

a. $\mathcal{W}$ is an $\mathbf{F}^{\bullet}$-partition of $X$;

b. $\mathcal{W}^{\prime}$ is a Whitney (a) partition of $V(f)$;

c. $\mathbf{0} \in T \in \mathcal{W}^{\prime}$ and $\operatorname{dim} T=1$;

d. for all $W \in \mathcal{W}\left(\mathbf{F}^{\bullet}\right)$ such that $W \nsubseteq V(f)$, for all $W^{\prime} \in \mathcal{W}^{\prime}$ such that $W^{\prime} \neq T,\left(W, W^{\prime}\right)$ satisfies the $a_{f}$ condition.

Then, the condition:

( $\dagger$ ) for all $W \in \mathcal{W}\left(\mathbf{F}^{\bullet}\right)$ such that $W \nsubseteq V(f),(W, T)$ satisfies the $a_{f}$ condition

is equivalent to all of the conditions in Corollary 4.18; in particular, it is equivalent to: there exists a non-zero linear form $\mathfrak{l}$ such that $\mathbf{0} \notin\left|\Gamma_{f, \mathfrak{l}}\left(\mathbf{F}^{\bullet}\right)\right|$.

Proof. Let $\mathcal{W}_{T}^{\prime}:=\left\{W^{\prime} \in \mathcal{W}^{\prime} \mid W^{\prime} \neq T\right\}$. By Corollary 3.9 of [18, our hypotheses imply that, if $p \in \mathcal{W}^{\prime} \in \mathcal{W}_{T}^{\prime}$, and $\mathfrak{l}^{\prime}$ is a non-zero linear form such that $V\left(\mathfrak{l}^{\prime}-\mathfrak{l}^{\prime}(p)\right)$ transversely intersects $W^{\prime}$ at $p$, then $p \notin \operatorname{supp} \phi_{\mathfrak{l}^{\prime}-\mathfrak{l}^{\prime}(p)}[-1] \psi_{f}[-1] \mathbf{F}^{\bullet}$.

Assume $(\dagger)$. By Corollary 3.9 of $\left[18, \psi_{f}[-1] \mathbf{F}^{\bullet}\right.$ is $\phi$-constructible with respect to $\mathcal{W}^{\prime}$. In particular, this implies that, if $\mathfrak{l}$ is a non-zero linear form such that $V(\mathfrak{l})$ transversely intersects $T$ at $\mathbf{0}$, then $\mathbf{0} \notin$ $\operatorname{supp} \phi_{\mathbf{r}}[-1] \psi_{f}[-1] \mathbf{F}^{\bullet}$. This implies Item 3 of Corollary 4.18

Assume Item 3 of Corollary 4.18 Fix a non-zero $\mathfrak{l}$ such that $V(\mathfrak{l})$ transversely intersects $T$ at $\mathbf{0}$ and $H^{*}\left(\phi_{\mathfrak{r}}[-1] \psi_{f}[-1] \mathbf{F}^{\bullet}\right)_{\mathbf{0}}=0$. As $\mathcal{W}^{\prime}$ satisfies Whitney (a), $V(\mathfrak{l})$ transversely intersects all of the strata of $\mathcal{W}^{\prime}$ in a neighborhood of $\mathbf{0}$. Thus, by the first paragraph of the proof, $\mathbf{0} \notin \operatorname{supp} \phi_{\mathfrak{r}}[-1] \psi_{f}[-1] \mathbf{F}^{\bullet}$. As $T$ is 1-dimensional, this, together with the first paragraph of the proof, implies that $\psi_{f}[-1] \mathbf{F}^{\bullet}$ is weakly $\phi$-constructible with respect to $\mathcal{W}^{\prime}$. By Corollary 3.9 of [18, this implies $(\dagger)$.

\section{References}

[1] Briançon, J., Maisonobe, P., and Merle, M. Localisation de systèmes différentiels, stratifications de Whitney et condition de Thom. Invent. Math., 117:531-550, 1994.

[2] Fulton, W. Intersection Theory, volume 2 of Ergeb. Math. Springer-Verlag, 1984. 
[3] Goresky, M. and MacPherson, R. Stratified Morse Theory, volume 14 of Ergeb. der Math. SpringerVerlag, 1988.

[4] Hamm, H. and Lê D. T. Un théorème de Zariski du type de Lefschetz. Ann. Sci. Éc. Norm. Sup., 6 (series 4):317-366, 1973.

[5] Hironaka, H. Stratification and flatness. In P. Holm, editor, Real and Complex Singularities, Oslo 1976, pages 199-265. Nordic Summer School/NAVF, 1977.

[6] Kashiwara, M. and Schapira, P. Sheaves on Manifolds, volume 292 of Grund. math. Wissen. SpringerVerlag, 1990.

[7] Lê, D. T. Calcul du Nombre de Cycles Evanouissants d'une Hypersurface Complexe. Ann. Inst. Fourier, Grenoble, 23:261-270, 1973.

[8] Lê, D. T. Topological Use of Polar Curves. Proc. Symp. Pure Math., 29:507-512, 1975.

[9] Lê, D. T. Some remarks on Relative Monodromy. In P. Holm, editor, Real and Complex Singularities, Oslo 1976, pages 397-404. Nordic Summer School/NAVF, 1977.

[10] Lê, D. T. Sur les cycles évanouissants des espaces analytiques. C. R. Acad. Sci. Paris, Sér. A-B, 288:A283-A285, 1979.

[11] Lê, D. T. and Saito, K. La constance du nombre de Milnor donne des bonnes stratifications. C.R. Acad. Sci., 277:793-795, 1973.

[12] Massey, D. Lê Cycles and Hypersurface Singularities, volume 1615 of Lecture Notes in Math. SpringerVerlag, 1995.

[13] Massey, D. Hypercohomology of Milnor Fibres. Topology, 35:969-1003, 1996.

[14] Massey, D. A Little Microlocal Morse Theory. Math. Ann., 321:275-294, 2001.

[15] Massey, D. Perverse Cohomology and the Vanishing Index Theorem. Top. and Appl., 125:299-313, 2002.

[16] Massey, D. Numerical Control over Complex Analytic Singularities, volume 778 of Memoirs of the AMS. AMS, 2003.

[17] Massey, D. Singularities and Enriched Cycles. Pacific J. Math., 215, no. 1:35-84, 2004.

[18] Massey, D. Vanishing Cycles and Thom's $a_{f}$ Condition. math.AG/0605369, 2006.

[19] Mather, J. Notes on Topological Stability. Notes from Harvard Univ., 1970.

[20] Parusiński, A. Limits of Tangent Spaces to Fibres and the $w_{f}$ Condition. Duke Math. J., 72:99-108, 1993.

[21] Sabbah, C. Proximité évanescente. Compos. Math., 62:283-328, 1987.

[22] Teissier, B. Cycles évanescents, sections planes et conditions de Whitney. Astérisque, 7-8:285-362, 1973. 